ARTICLE

\title{
Moderate levels of 5-fluorocytosine cause the emergence of high frequency resistance in cryptococci
}

Yun C. Chang (1) ${ }^{1 凶}$, Ami Khanal Lamichhane ${ }^{1}$, Hongyi Cai ${ }^{2}$, Peter J. Walter $\mathbb{D}^{2}$, John E. Bennett ${ }^{3} \&$ Kyung J. Kwon-Chung ${ }^{1}$

The antifungal agent 5-fluorocytosine (5-FC) is used for the treatment of several mycoses, but is unsuitable for monotherapy due to the rapid development of resistance. Here, we show that cryptococci develop resistance to 5-FC at a high frequency when exposed to concentrations several fold above the minimal inhibitory concentration. The genomes of resistant clones contain alterations in genes relevant as well as irrelevant for 5-FC resistance, suggesting that 5-FC may be mutagenic at moderate concentrations. Mutations in FCY2 (encoding a known permease for 5-FC uptake), FCY1, FUR1, UXS1 (encoding an enzyme that converts UDP-glucuronic acid to UDP-xylose) and URA6 contribute to 5-FC resistance. The uxs1 mutants accumulate UDP-glucuronic acid, which appears to down-regulate expression of permease FCY2 and reduce cellular uptake of the drug. Additional mutations in genes known to be required for UDP-glucuronic acid synthesis (UGD1) or a transcriptional factor NRG1 suppress UDP-glucuronic acid accumulation and 5-FC resistance in the uxs1 mutants.

\footnotetext{
${ }^{1}$ Molecular Microbiology Section, Laboratory of Clinical Immunology and Microbiology, NIAID, NIH, Bethesda, MD, USA. ${ }^{2}$ Clinical Mass Spectrometry Core, NIDDK, NIH, Bethesda, MD, USA. ${ }^{3}$ Laboratory of Clinical Immunology and Microbiology, NIAID, NIH, Bethesda, MD, USA. ${ }^{凶}$ email: ychang@niaid.nih.gov
} 
5

-fluorocytosine (5-FC), a fluorinated pyrimidine, is one of the oldest antifungals of use in treatment of cryptococcosis, as well as in candidiasis and chromoblastomycosis. It was synthesized in $1957^{1}$ and is the only commercially available compound that inhibits macromolecule synthesis in fungi for clinical use. 5-FC enters fungal cells through one or more permeases among which purine-cytosine permease, Fcy2, is the primary one known for uptake of the drug ${ }^{2-6} .5-\mathrm{FC}$ itself is not toxic and is converted to its metabolically active form 5-fluorouracil inside the cells by cytosine deaminase, Fcy1. 5fluorouracil can be further processed to 5-fluorouridine monophosphate or 5-fluorodeoxyuridine monophosphate and inhibit DNA replication, transcription, and protein synthesis $4,5,7,8$.

Currently, 5-FC in combination with amphotericin B remains the gold standard for induction therapy in cryptococcosis followed by maintenance therapy with fluconazole ${ }^{9}$. Combination therapy is known for more rapid cerebrospinal fluid sterilization and, though controversial, lower rates of treatment failure and improved mortality when compared with alternate regimens ${ }^{10,11}$. 5-FC, however, is not suitable for monotherapy due to rapid development of resistance in fungal pathogens. For instance, in the largest case series, only 10 of 23 (43\%) cryptococcosis patients responded to flucytosine monotherapy ${ }^{12}$. From the 13 failing patients, isolates were available for study from 12 patients, among which six isolates were highly resistant to 5 -FC. All six isolates had markedly decreased uptake of flucytosine and cytosine, suggesting an alteration in permease activity and five of them were cross resistant to 5 -fluorouracil suggesting an altered uracil phosphoribosyltransferase $\mathrm{e}^{13}$. However, this study was carried out before molecular technology was available and no subsequent study has been conducted on the clinically acquired 5-FC resistance in Cryptococcus spp. Resistance to 5-FC in other clinically relevant fungal species is known to emerge as a consequence of mutations primarily in the FCY2, FCY1, and FUR1 genes encoding the purine-cytosine permease, cytosine deaminase, and uracil phosphoribosyltransferase, respectively ${ }^{6,14-17}$. Many more genes were found to contribute to 5-FC resistance through chemogenomic analysis in Saccharomyces cerevisiae ${ }^{18}$ but the relevance of these genes in cryptococcal 5-FC resistance remains unclear. Recently, the UXS1 gene, known to encode an enzyme that converts UDP-glucuronic acid (UDP-GlcUA) to UDP-xylose for capsule biosynthesis, has been reported to play a role in cryptococcal resistance to 5 -FC in vitro ${ }^{19}$.

Here, we show that various strains of Cryptococcus neoformans and C. gattii species complex develop resistant clones at very high frequency at the drug concentrations 20-to 100-fold of the minimal inhibitory concentration (MIC). Furthermore, the resistance phenotype is unstable in many of these clones. An array of mutations including insertion, deletion, substitution, and variations in chromosome copy number are identified via genomic sequencing of the resistant clones. Interestingly, the 5-FC resistance phenotype in the uxs 1 deletion mutants is unstable and we identify two genes whose mutations can independently suppress the 5-FC resistance phenotype of the uxs1 mutants. We demonstrate that mutations in UXS1 and the presence of its suppressors affect the levels of intracellular UDP-GlcUA. Importantly, accumulation of UDP-GlcUA appears to modulate the expression levels of cytosine permease Fcy2 which controls the accumulation of 5-FC with an outcome of altered drug susceptibility.

\section{Results}

5-FC resistant colonies emerge at high frequency at concentrations several folds above the MIC. An interesting phenomenon was observed when we examined the 5-FC susceptibility in the strains of Cryptococcus species complex. The 5 -FC resistant colonies emerged with frequency near $1 \times 10^{-1}$ at concentrations close to 20 -fold above the MIC of H99, a patient isolate of C. neoformans VNI strain ${ }^{20}$, R265 a Vancouver outbreak strain of C. gattii VGIIa ${ }^{21}$, and WM276, a VGI reference strain of C. gattii isolated from Australian environment ${ }^{22}$ (Fig. 1 and Supplementary Fig. 1). In each case, the frequency of resistance decreased dramatically at higher concentrations of the drug. Since all cryptococci thus far tested is known to develop unstable heteroresistance to fluconazole at high frequency ${ }^{23-25}$, we determined if similar phenomena occurred with 5-FC. We randomly selected 15 each of large- and small-sized resistant colonies grown on 5-FC agar plates in which the resistance frequency was greater than $1 \times 10^{-3}$. The randomly selected clones were cultured in the nonselective YPD medium and transferred daily to fresh media. The frequency of 5-FC resistant population in each strain was determined periodically during the passage period. Close to 50\% of the clones derived from the large-sized 5-FC resistant colonies remained resistant to the drug at the end of transfers in all three strains indicating that resistance to 5-FC in those clones were relatively stable (Supplementary Fig. 2, left panels). Considerably fewer number of clones derived from small-sized resistant colonies remained resistant at the end of passage. Furthermore, many of the unstable clones rapidly lost their resistance in less than seven passages regardless of their original colony size on the 5-FC media. These observations indicated that the stability of resistance
H99

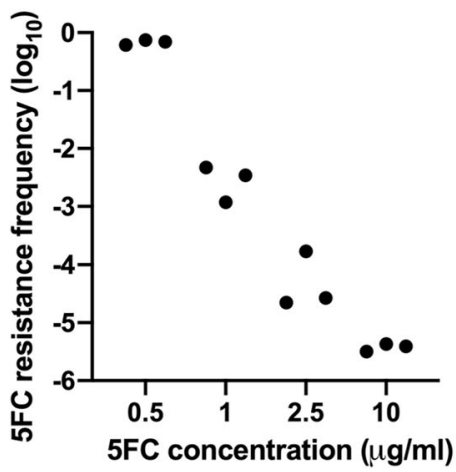

R265

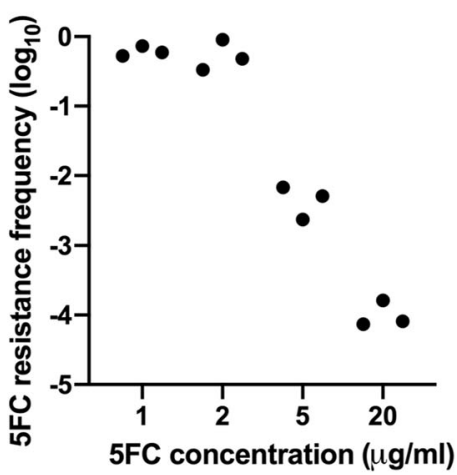

WM276

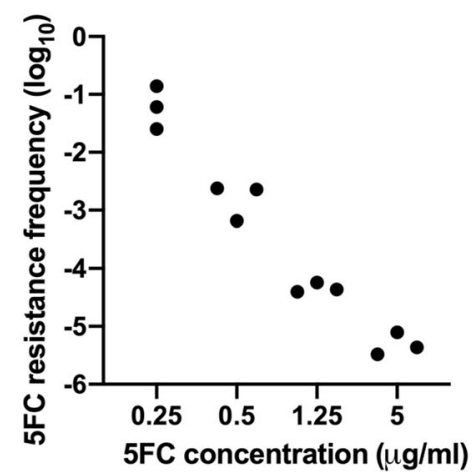

Fig. 1 The frequency of 5-FC resistance varies according to 5-FC concentrations. Cells from H99, R265, and WM276 were plated on YNB media supplemented with indicated amounts of 5-FC which were approximately corresponding to 20-, 40-, 100-, and 400-fold of 5-FC MIC of each tested strain. Plates were incubated at $30^{\circ} \mathrm{C}$ for 7 days and photographed. The frequency of 5-FC resistance was calculated by dividing the number of the 5-FC resistant colonies by the total number of the input cells. The experiments were repeated three times. 
H99

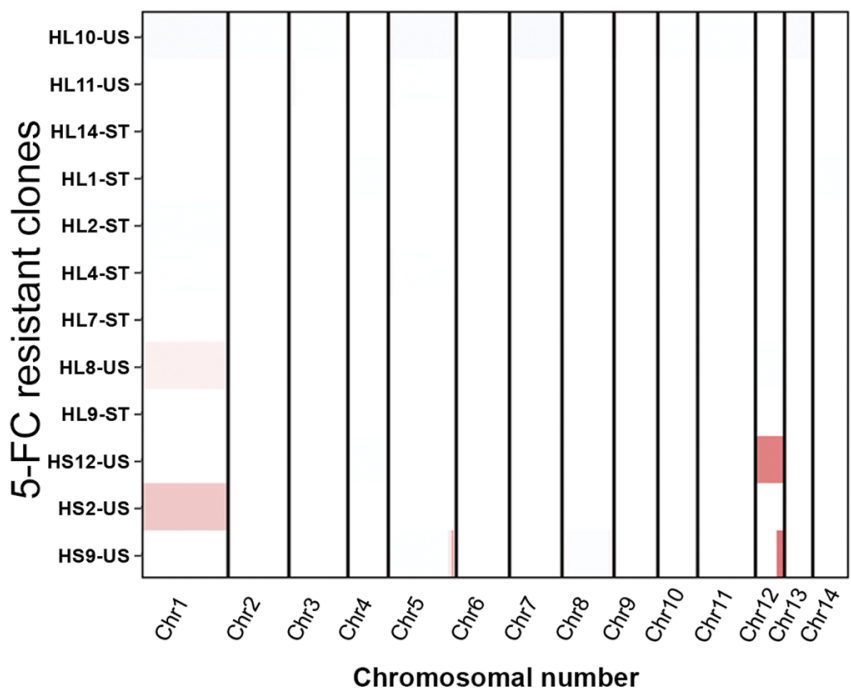

R265
WM276

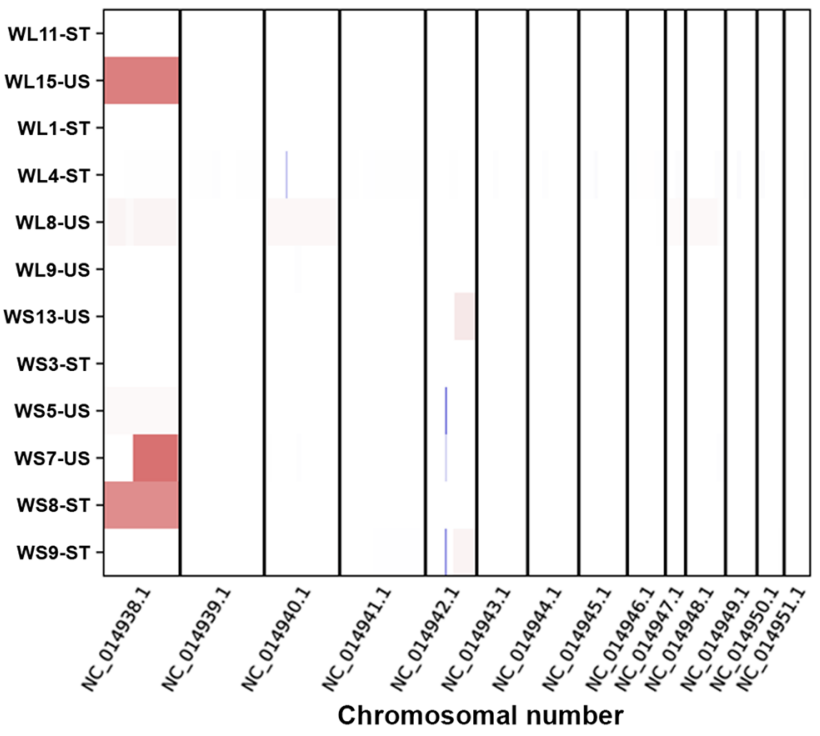

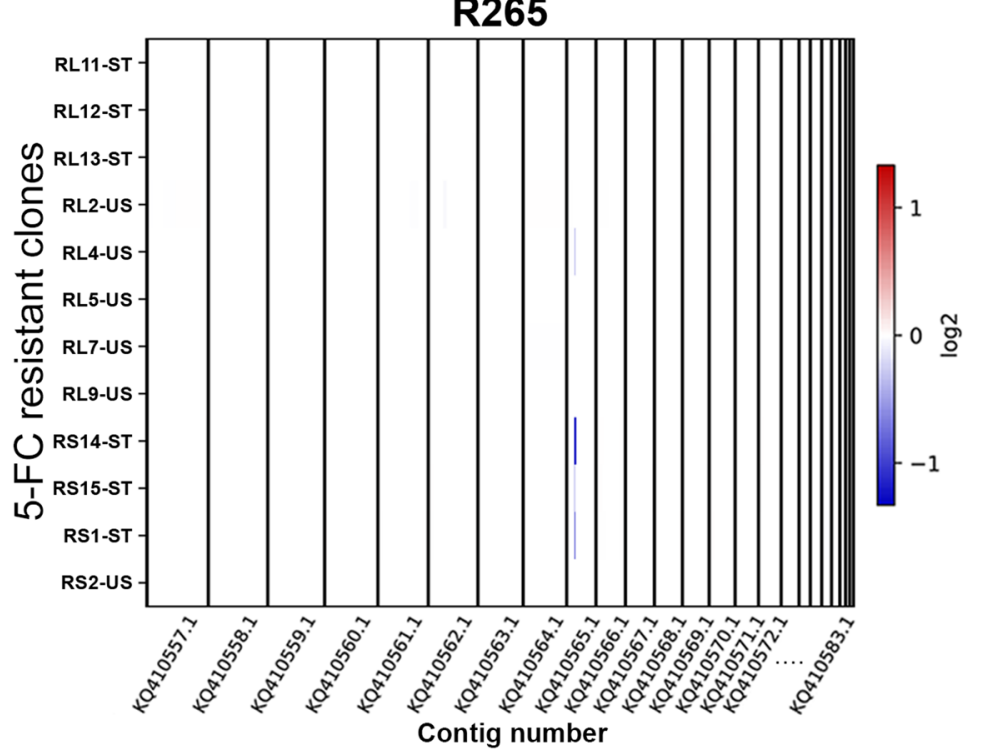

Fig. 2 Chromosome copy number analysis. The genomic sequences of twelve 5-FC resistant clones each from H99, R265, and WM276 were determined, and the copy number of each chromosome was calculated. The change of copy number is represented by the intensity of the color. The red color represents copy gain and blue color represents copy loss in units of log2 as shown by the color scale. The color bar represents the location of copy number change in the chromosome. The name of chromosome or contig is indicated at the bottom of each panel and the width of the column represents the size of chromosome or contig. The name of each clone is on the left side of each panel. The " $\mathrm{L}$ " and " $\mathrm{S}$ " in the name represent the size of the colony at the time of its isolation and ST and US in the suffix indicates the stability of each 5-FC resistant clone as either stable or unstable, respectively.

in the 5-FC resistant clones are exceedingly greater than the fluconazole heteroresistant clones in Cryptococcus species.

Genomic sequencing of the 5-FC resistant clones. It has been shown that the fluconazole heteroresistant clones derived from H99 and other clinical isolates are unstable aneuploid ${ }^{25,26}$. We performed whole-genome sequencing to determine if aneuploidy had occurred among the 5-FC resistant clones. We arbitrarily selected six each of stable and unstable 5-FC resistant clones derived from the three strains. The frequency of drug-resistant progeny at the end of daily transfer was greater than $90 \%$ in the stable clones and it was less than $7 \%$ in the unstable clones. We detected only a few clones had changes in the chromosome copy number. HL8, HS2, and HS12 were derived from H99 and had a slight increase in copy number of chromosome 1 (chrl) or chr12 and HS9 had an increase in a segment of chr12 (Fig. 2). Two WM276 derived clones, WL15, and WS8, had increased copy number of chr1 and WS7 had an increase in a segment of chr1. No clear increase in chromosome copy number was detected in clones derived from R265 except that two clones, RS14 and RS1, had a reduction of the copy number in a segment of contig KQ410565.1. Among the seven clones with a clear increase in chromosome copy number, only one clone, WS8, was stable while the rest of the clones lost their resistance during the transfers. These data suggest that the increase in chromosome copy number may contribute to the 5-FC resistance in some but not all of the 5$\mathrm{FC}$ resistant clones.

Chr1 duplication contributes to 5-FC resistance in H99. Since the copy number of chr1 was elevated in HL8 and HS2, chr1 
a

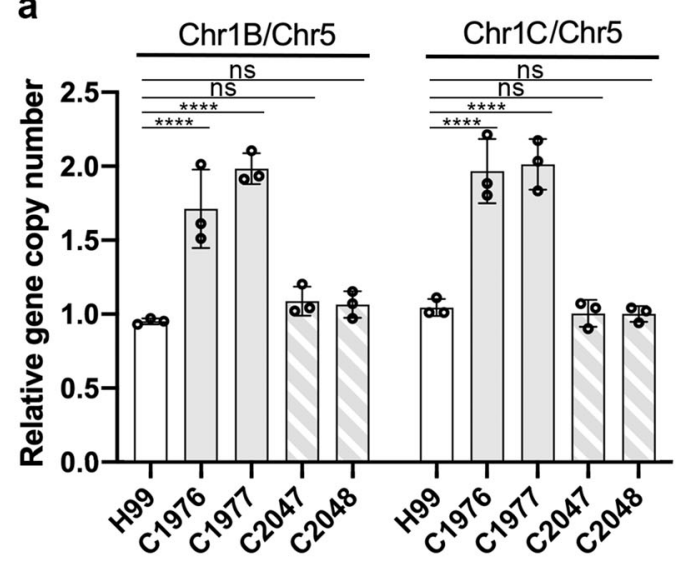

C

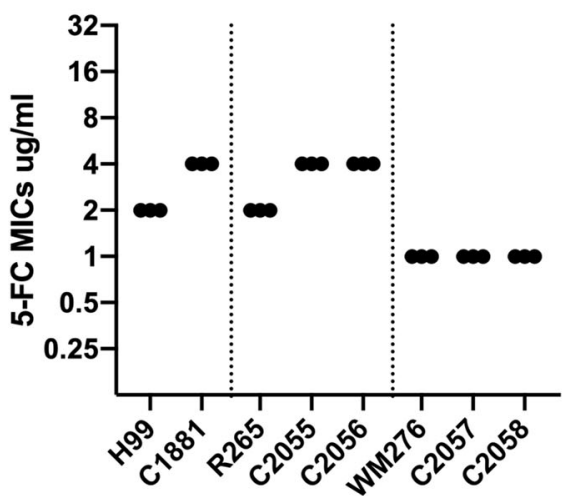

b

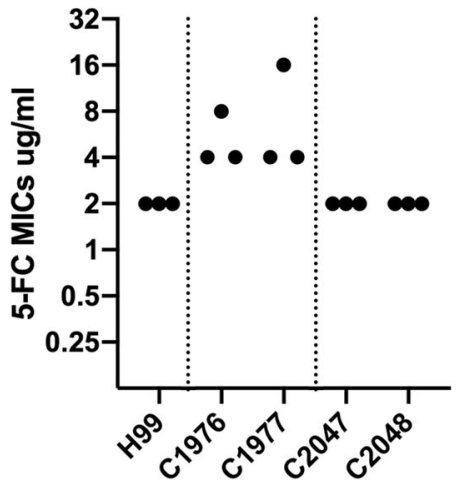

d

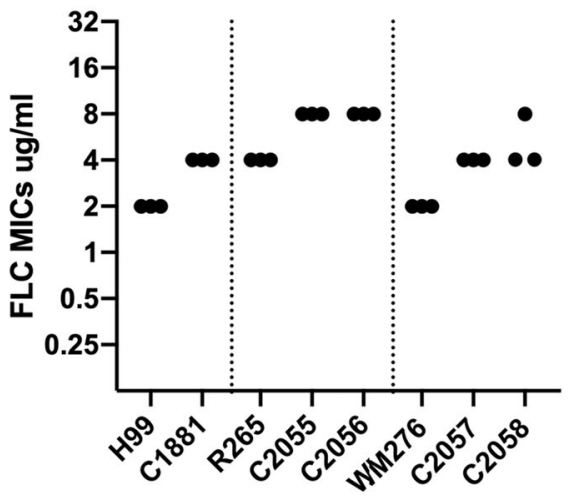

Fig. 3 Chr1 duplication and extra copy of AFR1 affects the 5-FC resistance levels. a Relative copy number of genes resided on chr1. Probes specific for the genes resided on the left or right arms of chr1 (Chr1B or Chr1C) were chosen to assess the changes in chr1 copy number. The PCR results of the chr1specific probes were compared to that of chr5, which served as unduplicated internal control in the indicated strains. Data are presented as mean values \pm standard deviation from three biological repeats of each strain. Two-way ANOVA with Tukey's test for multiple comparisons (ns $=$ not significant, ${ }^{\star \star \star \star}=p$ $<0.0001$ ). b Broth 5-FC MIC of the strains containing duplicated chr1 and the strains after repeated transfers which lost the duplicated chr1. $\mathbf{c}$ Broth 5-FC MIC of the strains containing extra copies of AFR1. d Broth fluconazole MIC of the strains containing extra copies of AFR1. The MIC determination was repeated three times for each strain.

aneuploidy was presumed to have contributed to 5-FC resistance. This assumption was based on the observation that clones heteroresistant to $32 \mu \mathrm{g} / \mathrm{ml}$ fluconazole invariably contained duplicated $\operatorname{chr} 1^{25}$. We generated two H99 derived heteroresistant clones, C1976 and C1977 (Supplementary Table 1), which were presumed to be disomic for chr1 and used them to determine the effect of chr1 duplication for 5-FC resistance. To assess the status of chr1 in C1976 and C1977, we analyzed the copy number of the genes located on each arm of chr1. The copy number of chr1 genes was 2 -fold of the genes located on chr5, confirming duplication of chr1 in C1976 and C1977 (Fig. 3a). After repeated passages of C1976 and C1977 in drug-free YPD media, we isolated clone C2047 and C2048, respectively. The copy number of the chr1 genes in C2047 and C2048 was similar to the wildtype H99 (Fig. 3a) indicating that the extra copy of chr1 in C1976 and C1977 was lost during the repeated transfer. Notably, the 5-FC MIC of C1976 and C1977 by broth microtiter testing was 4-16 $\mu \mathrm{g} / \mathrm{ml}$ compared to $2 \mu \mathrm{g} / \mathrm{ml}$ in $\mathrm{H} 99$ or the repeated passage strains, C2047 and C2048 (Fig. 3b). These results indicated that chr1 duplication contributed moderately to the 5-FC resistance though the mechanism remains unknown. Since chrl contains $A F R 1$, an ABC transporter gene important for fluconazole resistance $^{27}$, we inserted an extra copy of $A F R 1$ in the chr3 of the wild-type strain $\mathrm{H} 99$ to assess if two copies of AFR1 gene could increase the 5-FC resistance levels. The 5-FC resistance levels in the resulting strain, C1881, were 2 -fold higher than that in
H99 suggesting that AFR1 in H99 plays a role in 5-FC resistance (Fig. 3c). Similarly, extra copies of AFR1 in R265 derived clones, C2055 and C2056, exhibited slightly increased levels of resistance to 5 -FC as well as fluconazole (Fig. 3c). In contrast, extra copies of AFR1 in WM276 derived clones, C2057 and C2058, displayed increased levels of resistance only to fluconazole but not to 5-FC (Fig. $3 \mathrm{c}$ and d). These results indicated that the role of AFR1 in 5FC resistance is not consistent but strain dependent in Cryptococcus species complex.

Identification of genetic variants in the genome of 5-FC resistant clones. Since the majority of 5-FC resistant clones showed no clear variation of copy number in the genome, we performed variant analysis of the 36 sequenced genomes to explore the possible existence of any other alterations. Interestingly, an array of genetic variants was found among the resistant clones, including single nucleotide variant, insertion, deletion, and substitution (Supplementary Data 1). We focused our analysis only on the variants in the exon regions of the annotated genes. Several variants were found in the exon regions of FCY1, FCY2, and FUR1 (Table 1). Two variants of UXS1, a recently identified gene important for 5 -FC resistance ${ }^{19}$, were found in WM276 derived clones, WL8 and WL9. Additionally, variants were detected in genes encoding a putative uridylate kinase, an epsilon DNA polymerase, a putative monosaccharide transporter, 
Table 1 Variants detected in the 5-FC resistance clones.

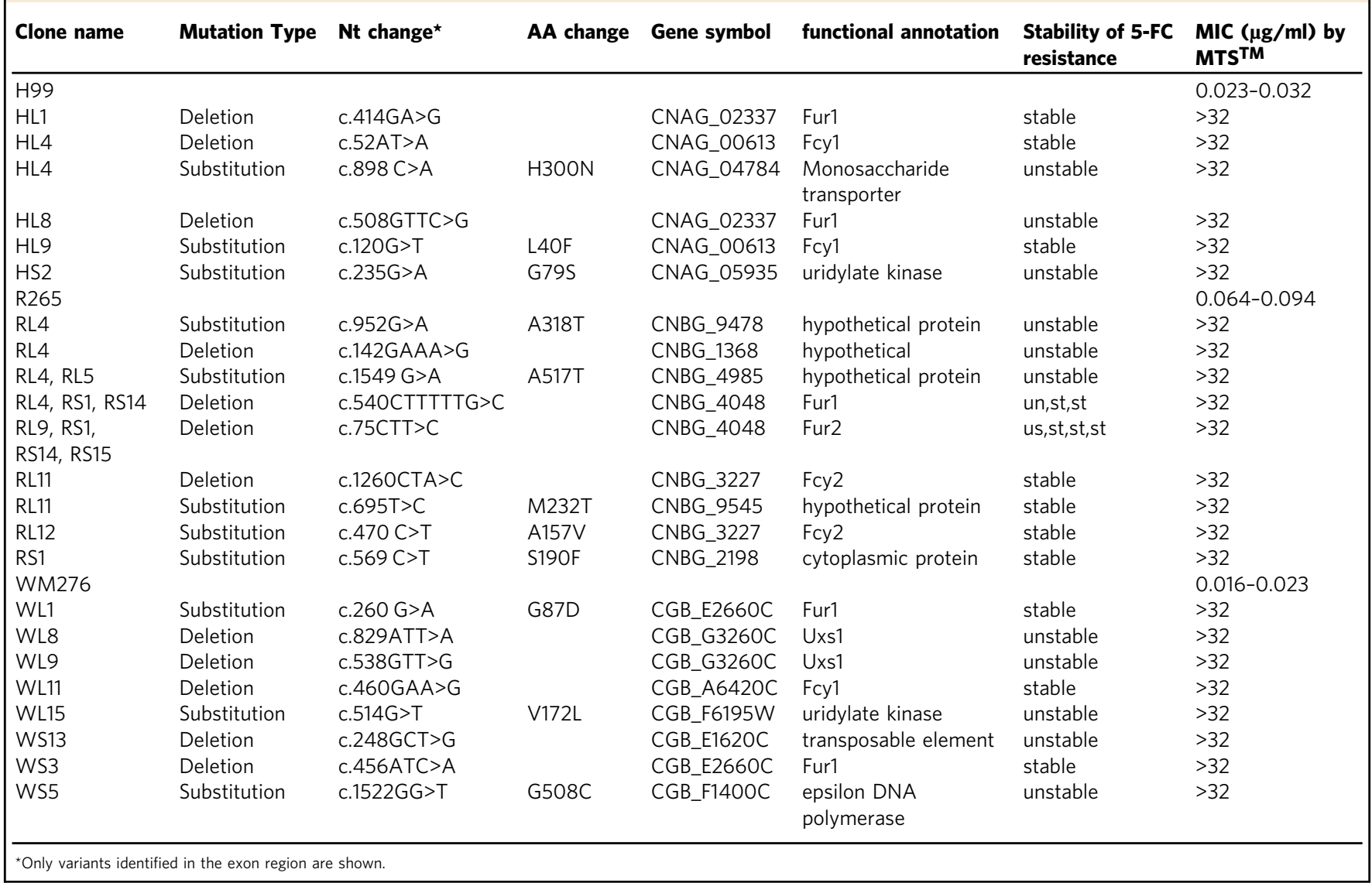

transposable elements, hypothetical proteins, and cytoplasmic proteins.

Verification of the genes important for 5-FC resistance. Before confirming the role of the variants containing genes in 5-FC resistance, we measured the levels of resistance to 5-FC in the 36 genome sequenced clones by MTSTM MIC test strips. All 36 clones appeared to be resistant to the highest concentration of 5FC on the test strips, $32 \mu \mathrm{g} / \mathrm{ml}$ (Table 1). Since all of the sequenced clones contained more than one genomic variant (Supplementary Data 1), we constructed deletion mutants or generated the same variants postulated to be responsible for 5-FC resistance in the wild type strains.

First, we focused on the importance of FCY1, FCY2, and FUR1 in 5-FC resistance and constructed deletion mutants of each gene in H99, R265, and WM276. Although FCY1, FCY2, and FUR1 are known to play a critical role in 5-FC resistance in other fungi ${ }^{6,15}-17$, their roles have not been unequivocally substantiated for cryptococcal 5-FC resistance ${ }^{28}$ except that FCY2 in R265 and FUR1 in $\mathrm{H} 99$ have been shown to be important for 5-FC resistance ${ }^{19,29}$. Table 2 shows that the deletion mutant of each of the FCY1, FCY2, and FUR1 gene derived from all three strains was

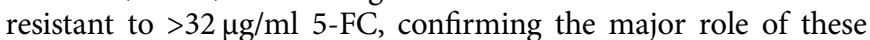
three genes in cryptococcal susceptibility to 5-FC as in other fungi.

Variants were detected in two putative essential genes encoding uridylate kinase (URA6; CGB_F6195W) and epsilon DNA polymerase (DPB2; CGB_F1400C) in the WM276 derived clones WL15 and WS5 (Table 1). Ura6 catalyzes the seventh enzymatic step in the de novo biosynthesis of pyrimidines which converts uridine monophosphate into uridine-5'-diphosphate in S. cerevisiae ${ }^{30}$. $D B P 2$ encodes the second largest subunit of DNA polymerase II and is required for the maintenance of chromosomal replication fidelity $^{31}$. To determine the importance of URA6 and DBP2 in 5-FC resistance, we generated the same variants for the two putative essential genes in the wild type strain WM276 by molecular manipulations through homologous integration. The resulting strain C2080 containing URA6 ${ }^{\text {V172L }}$ variant displayed markedly higher MIC than the wild type $(0.19-0.38 \mu \mathrm{g} / \mathrm{ml}$ vs. $0.016-0.023 \mu \mathrm{g} /$ ml) (Table 2). This indicated that URA6 contributes to 5-FC susceptibility which has not been implicated in other fungi. However, C2080 was not as resistant as WL15, the original carrier of URA6 ${ }^{V 172 L}$ variant (MIC $>32 \mathrm{mg} / \mathrm{ml}$ Table 1$)$, suggesting that additional unverified changes might have rendered WL15 to be more resistant to 5-FC than C2080. In contrast, the $D P B 2^{G 508 C}$ variant containing strain, $\mathrm{C} 2171$, had similar MIC as the wild-type WM276 (Table 2) indicating that the DPB2 ${ }^{G 508 C}$ variant was not involved in 5-FC resistance and other unverified changes in WS5 might be responsible for its resistance.

HL4 was a H99 derived clone that produced a large-sized resistant colony that contained two variants, a deletion variant in FCY1 encoding region and a substitution variant, $\mathrm{H} 300 \mathrm{~N}$, in the monosaccharide transporter CNAG_04784 (Table 1). Since importance of FCY1 in 5-FC resistance has been confirmed, we obtained a CNAG_04784 deletion mutant, 32C12, from the Fungal Genetics Stock Center to determine the importance of CNAG_04784. The MIC of 32C12 was similar to the wild-type strain (Table 2) indicating that the $\mathrm{H} 300 \mathrm{~N}$ variant in CNAG_04784 of HL4 was unrelated to 5-FC resistance and suggested that the single nucleotide deletion in FCY1 caused 5-FC resistance in HL4.

RS1 was a small resistant colony derived from R265 that contained a S190F variant in a cytoplasmic protein encoding 
Table 2 MIC determined by Liofilchem ${ }^{\circledR}$ MIC Test Strips ${ }^{\mathrm{TM}}$.

Strain Description

5-FC MIC $(\mu \mathrm{g} / \mathrm{ml})$

Strains containing gene deletion or substitution

H99 derived strains

$\mathrm{H} 99$

C1918

wild type

fcy $1 \Delta$

fcy $2 \Delta$

fur1s

C1920

$32 \mathrm{C} 12$

CNAG_04784A

$n r g 1 \Delta$

$0.023-0.032$

$>32$

$>32$

$>32$

$0.032-0.047$

$0.032-0.047$

R265 derived strains

R265

C1960

C1911

C2178

wild type

fcy $1 \Delta$

fcy $2 \Delta$

furr $1 \Delta$

C2172

CNBG_21984

WM276 derived strains

WM276

C1832

wild type

fcy $1 \Delta$

fcy $2 \Delta$

fur7s

C1900

C2022

C2080

C2171

44 transfers of $\mathrm{C} 1900$

URA6V172L

$D P B 2^{G 508 C}$

Strains related to suppressor

KN99 derived

strains

KN99

$13 \mathrm{C} 2$

C1952

C2050

C2119

C2133

C2168

WM276 related strain

C2053

C2074

C2104

C2072

C2113

C2061

C2151

C2152

C2153

WL8 derived strains

WL8

C1933

C2028

C2143

WL9 derived strains

WL9

C1943

C2029

C2030

C2071

C2111

wild type
uxs1
47 transfers of $13 C 2$
Knrg1 in $13 C 2$
Kugd1 in 13C2
Knrg1 in KN99
Kugd1 in KN99

Kugd1 in KN99

0.064-0.094

$>32$

$>32$

$>32$

$0.064-0.125$

$0.016-0.023$

$>32$

$>32$

$>32$

$>32$

$0.19 / 0.38$

$0.012-0.016$

uxs1 $1 \Delta>32$

UXS1 complemented $\quad 0.023$

Wugd1 in uxs14 $\quad 0.047-0.064$

Wnrg1 in uxs14

$>32$

Xnrg1 in uxs14

Wugd1 in WM276

Fcy2-mNG in WM276

$>32$

$0.012-0.023$

$0.032-0.047$

$>32$

Fcy2-mNG in uxs14 + Wugd1 0.047-0.064

WM276 derived $\quad>32$

51 transfers of WL8 $\quad 0.016-0.023$

$B C K 7^{R 1382^{*}}$ in WL8 >32

Wugd1 in WL8

0.016-0.023

WM276 derived

51 transfers of WL9

$>32$

$0.032-0.047$

$>32$

Wugd1 in WL9

$0.032-0.047$

$>32$

$>32$

Identification of the uxs1 suppressors. It was interesting to find that the UXS1 gene in the clones WL8 and WL9 derived from WM276 was partially deleted and yet their 5-FC resistance phenotype was unstable (Table 1). Similarly, the FUR1 gene was found partially deleted in both HL8 and RL4 clones derived from H99 and R265 respectively and their resistance phenotype was unstable. Because it is known that deletion of $U X S 1^{19}$ or FUR1 causes resistance to 5-FC (Table 2), we thought that the instability of these clones could be due to the development of suppressors during repeated transfers in the nonselective medium. Since all four strains contained additional variants in the genome (Supplementary Data 1), we used the strains containing simple deletion of UXS1 in KN99a (strain 13C2) or FUR1 in WM276 (strain C1900) and repeated the transferring experiment to test the stability of their 5-FC resistance. Both $13 \mathrm{C} 2$ and $\mathrm{C} 1900$ were resistant to $>32 \mu \mathrm{g} / \mathrm{ml} 5$-FC but only the uxs 1 mutant (13C2) was unstable and lost resistance after repeated transfers (Table 2, $13 \mathrm{C} 2$ vs. C1952 and C1900 vs. C2022). Since the 5-FC resistance phenotype was consistently unstable in all three different types of uxs1 deletion mutants, WL8, WL9, and 13C2, we focused our analysis on the $u x s 1$ mutants to identify the possible suppressors. We randomly selected and sequenced the genomes of five clones each derived from the three different uxs1 strains that lost the resistance after repeated transfers. In all five clones derived from $13 \mathrm{C} 2$, we identified a same deletion variant at the C-terminal end of CNAG_05222 which encodes a transcriptional regulator Nrg1 (Supplementary Data 2). Additionally, in all the five clones derived from WL9, we identified a missense variant of CGB_D0330C which encodes a UDP-glucose 6-dehydrogenase Ugd1, and a nonsense variant of CGB_F0330C which encodes a RAN protein kinase Ran 1 . While we found no commonly shared variants among the five WL8 derived clones, a missense variant of UGD1 in four and a nonsense variant of CGB_I2500W which encodes a mitogen-activated protein kinase kinase kinase Bck1 in three among the WL8 derived clones (Supplementary Data 2).

To verify the importance of the identified variants in suppression of the uxs 1 -induced 5-FC resistance, we regenerated the same type of variants in the original uxs 1 mutants, $13 \mathrm{C} 2$, WL8, and WL9 by molecular manipulations. When the native NRG1 gene in $13 \mathrm{C} 2$ was replaced by the NRG1 deletion variant, Knrg1, the resulting clone lost its resistance (Table 2, C2050). This result indicated that the $n r g 1$ variant could function as a suppressor for uxs1 in KN99a background. When the UGD1 $1^{G 19 A}$ variant, Wugd1, was introduced into WL9, the resistance phenotype was suppressed (Table 2, C2030) but the $R A N 1^{Y 45^{*}}$ variant failed to suppress the phenotype (Table 2, C2029) suggesting that only the UGD1 $1^{G 19 A}$ variant was responsible for suppression of the resistance in WL9 derived clones. In contrast, the $B C K 1^{R 1382^{*}}$ variant failed to suppress the 5 -FC resistance phenotype in WL8 (Table 2, C2028). Although we did not examine the function of $U G D 1^{I 54 F}$ variant detected in the WL8 derived clones, the UGD1 ${ }^{G 19 A}$ variant found in WL9 derived clones was able to suppress the 5-FC resistance in WL8 (Table 2, C2143). We also generated a uxs1 deletion mutant, C2053, in WM276 background. C2053 was resistant to $>32 \mu \mathrm{g} / \mathrm{ml}$ 5 -FC and when the uxs $1 \Delta$ mutation was complemented, the resulting strain lost its resistance (Table 2, C2074). Furthermore, when the UGD1 $1^{G 19 A}$ variant was introduced into C2053, the resulting strain lost its resistance (Table 2, C2104). These data indicated that the $U G D 1^{G 19 A}$ variant could function as a suppressor in all three types of usx 1 deletion mutants in WM276 background.

Although WM276 (C. gattii) and KN99a (C. neoformans) belong to closely related species, numerous differences were found in the sequences of UGD1 and NRG1 between the two strains. To determine if the identified variants could function as suppressors in 
a

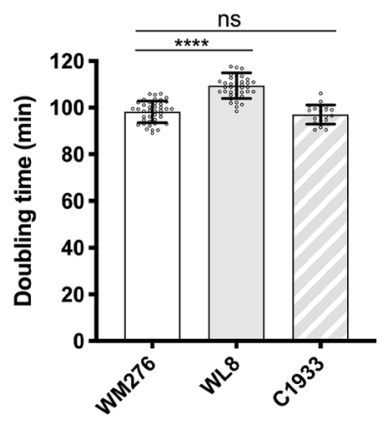

b

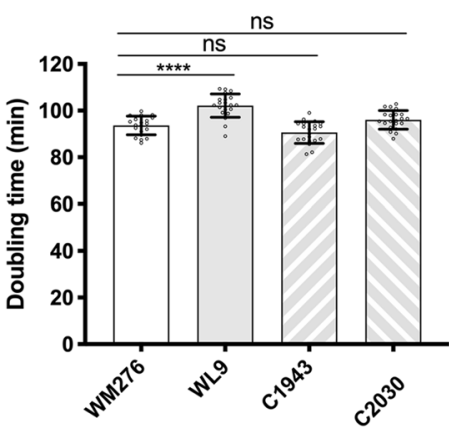

C

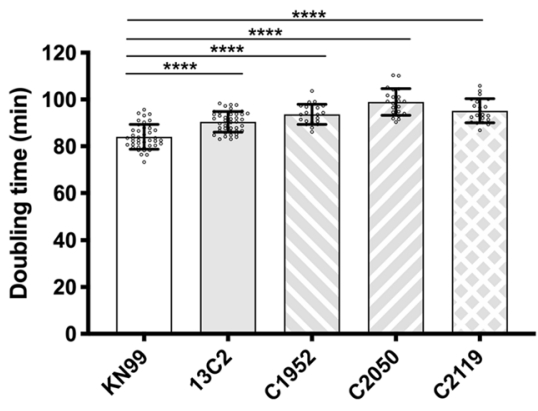

Fig. 4 Growth rate is different between the uxs1 suppressor strains in C. neoformans and C. gattii. The doubling time of indicated strain is determined using a plate reader. a C1933 is a 5-FC sensitive strain derived from repeated transfers of WL8. b C1943 is a 5-FC sensitive strain derived from repeated transfers of WL9. C2030 is a strain containing UGD1G19A that was manually introduced into WL9. c C1952 is a 5-FC sensitive strain derived from repeated transfers of 13C2. C2050 and C2119 are suppressor strains obtained by molecular manipulations which contained Knrg1 and UGDG19A, respectively. The experiments were repeated at least three times $(n \geq 21)$ and data are presented as mean values \pm standard deviation. One-way ANOVA with Tukey's test for multiple comparisons ( $\mathrm{ns}=$ not significant, and ${ }^{\star \star \star *} p<0.0001$ ).

both species, we generated the same type of UGD1 and NRG1 variants based on the sequences of WM276 and KN99a respectively. The UGD1 ${ }^{G 19 A}$ variant designed according to KN99a, Kugd1, was able to suppress the 5-FC resistance in the $13 \mathrm{C} 2$, a uxs $1 \Delta$ mutant of KN99a (Table 2, C2119). This indicates that UGD1G19A variant originally detected in WM276 (C. gattii) derived clones could also function as a suppressor of uxs 1 in $C$. neoformans. However, the partially deleted NRG1 variant designed according to WM276, Wnrg1, failed to suppress the 5-FC resistance in uxs1 14 , C2053, and WL9 derived from WM276 (Table 2, C2072 and C2071). Since the $\mathrm{N}$-terminal sequence of Nrg1 is conserved between WM276 and KN99 $a$ but the C-terminal sequence is diverged, we constructed a mosaic nrg1 which contained the wild type NRG1 of WM276 in the $5^{\prime}$-end and the NRG1 deletion variant of KN99 $\alpha$ in the $3^{\prime}$-end. The resulting construct, $X n r g 1$, still failed to suppress the 5-FC resistance phenotype of uxs1 mutants in WM276 background (Table 2, C2113 and C2111) indicating that the NRG1 deletion variant only functioned as a suppressor in C. neoformans but not in C. gattii.

The growth rate is different among different uxs1 suppressor strains. We suspected that the growth rate of WL8, WL9, and 13C2 had been retarded due to the uxs1 mutations and cells with faster growth rate could have been selected out through repeated passages in drug-free media and became dominant at the end of the experiments. Figure 4 shows that the doubling time of WL8 and WL9 was significantly longer than that of WM276 which indicates that the growth rate had been retarded in the WM276 uxs 1 mutants. In contrast, the doubling time of clones derived from WL8 and WL9 after repeated transfers, C1933 and C1943, respectively, was significantly shorter than the parental strains and was similar to the wild type WM276 (Fig. 4a and b). Furthermore, introducing UGD1 $19 A$ into WL9 also produced a strain with similar doubling time as the wild type (Fig. 4b, C2030). These data indicated that repeated transfer of the C. gattii uxs1 mutants in the drug-free medium was selective for cells with faster growth rate. Surprisingly, although the doubling time of 13C2 was significantly longer than KN99a, the doubling time of C1952 generated from the repeated transfers of 13C2 was similar to $13 \mathrm{C} 2$ and was significantly longer than KN99a (Fig. 4c). Therefore, repeated transfer of KN99a uxs1 $1 \Delta$ mutant in the rich medium did not select for cells with growth rate advantage. Furthermore, manual introduction of the Knrg1 or UGD1G19A variant into $13 \mathrm{C} 2$ did not improve its growth rate (Fig. 4c, C2050 and C2119). These results indicated that in C. neoformans, the uxs $1 \Delta$ suppressors fail to improve the growth rate defect of the uxs $1 \Delta$ mutant.

5-FC susceptibility is correlated with the UDP-GlcUA levels in the cells. It has been shown that the uxs $1 \Delta$ mutant of $C$. neoformans accumulates high amounts of nucleotide sugar UDP-GlcUA ${ }^{32}$ and accumulation of UDP-GlcUA has been proposed to suppress the toxicity of $5-\mathrm{FC}^{19}$. We measured the levels of nucleotide sugars in the cells to determine if the suppressors of the uxs 1 mutants could modulate the levels of UDP-GlcU. Figure $5 \mathrm{a}$ shows that UDP-GlcUA concentration was significantly higher in the uxs 1 deletion mutants C2053, WL8, and WL9 derived from WM276 indicating that $C$. gattii uxs $1 \Delta$ mutants accumulate high levels of UDP-GlcUA as is the case in C. neoformans ${ }^{32}$. Introduction of the $U G D 1^{G 19 A}$ variant into either of the three uxs 1 deletion mutants, C2053, WL8, and WL9, significantly reduced the concentration of UDP-GlcUA in the cells (Fig. 5a. C2104, C2143, and C2130, respectively). Similarly, the uxs $1 \Delta$ mutant of $\mathrm{KN} 99 a, 13 \mathrm{C} 2$, accumulated significantly higher amounts of UDP-GlcUA compared to that of the wild type KN99a (Fig. 5b). Also, C2050 and C2119, which contained either the $n r g 1$ deletion variant or the UGD1 ${ }^{G 19 A}$ variant in $13 \mathrm{C} 2$, synthesized similar amounts of UDP-GlcUA as the wild type (Fig. 5b). These results indicated that the uxs $1 \Delta$ mutants of both cryptococcal species accumulate high levels of UDP-GlcUA and the accumulation is diminished in the suppressor containing strains.

The Ugd1 function or the UGD1 expression levels is reduced in suppressor containing strains. We have identified two types of genomic suppressors in uxs $1 \Delta$ mutants. One is mutation in UGD1 and the other is genetic alteration in NRG1. UGD1 and UXS1 are functionally related genes for capsule synthesis in $C$. neoformans ${ }^{32-35}$. UGD1 encodes a UDP-glucose 6-dehydrogenase which converts UDP-glucose to UDP-glucuronic acid ${ }^{32}$. UXS1 is downstream of UGD1 and encodes an enzyme that converts UDP-GlcUA to UDP-xylose ${ }^{35}$. It is known that loss-of-function mutations in upstream components of a pathway can prevent the accumulation of the compound caused by mutation of the downstream gene and form suppression. For example, in S. cerevisiae, deletion of $A D E 13$ leads to accumulation of a toxic metabolite (S)-2-[5-Amino-1-(5-phospho-D-ribosyl)-imidazole4-carboxamido]succinate and causes a growth defect, which can be suppressed by loss of upstream pathway components ${ }^{36}$. Unlike the loss-of-function mutations in Ade13 suppressors, the 
a

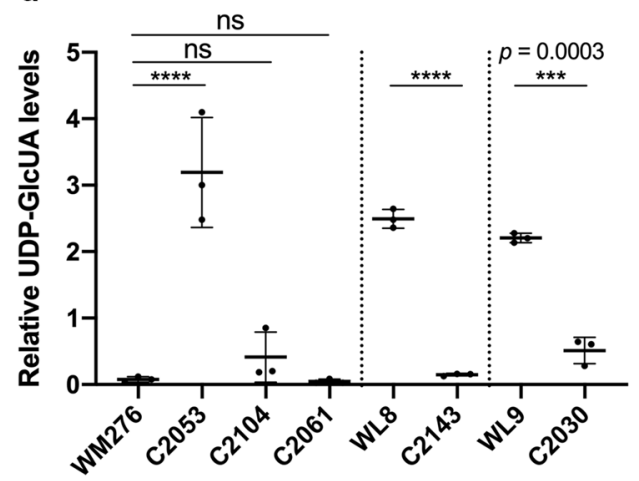

b

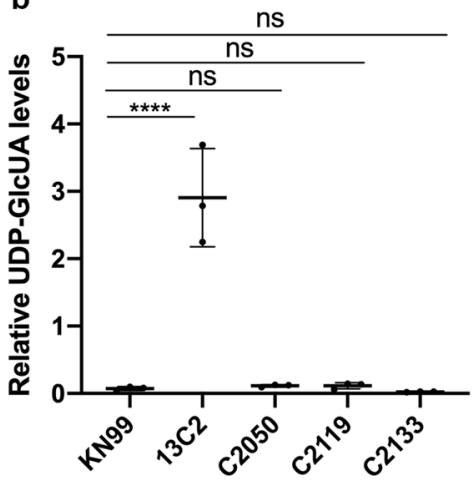

Fig. 5 The levels of UDP-glucuronic acid are high in the uxs1 mutants and low in the suppressor strains. Nucleotide sugars were analyzed as described under "Methods". Relative amounts of UDP-glucuronic acid were determined in strains derived from WM276 (a) and KN99 $\alpha$ (b). The experiments were repeated three times and data are presented as mean values \pm standard deviation. One-way ANOVA with Tukey's test for multiple comparisons (ns $=$ not significant, ${ }^{\star \star *} p<0.0002$, and $\left.{ }^{\star \star \star \star} p<0.0001\right)$.

$U G D^{G 19 A}$ variant is functional in terms of producing UDPGlcUA (Fig. 5). However, the activity of mutant protein may have been suffered since the amounts of UDP-GlcUA in the suppressor strain of the uxs $1 \Delta$ mutants was not significantly higher than that of the wild type. In addition, while the ugd1 $1 \Delta$ mutant was acapsular, the $U G D^{G 19 A}$ containing strains were encapsulated in both KN99 $\alpha$ and WM276 backgrounds (Fig. 6a). But the capsule size in the $U G D^{G 19 A}$ containing strains was slightly smaller than that of the wild type in both KN99 and WM276 suggesting that $U G D^{G 19 A}$ variant may have lost some of its functions (Fig. 6c). The second type of uxs 1 suppressor, NRG1, was found partially deleted in KN99a. NRG1 encodes a transcription factor that has been shown to regulate the expression of $U G D 1^{37}$. It is possible that the action of suppression by Knrgl is through downregulation of UGD1 since we found the expression levels of UGD1 were reduced in $K n r g 1$ containing strains compared to the wild type (Fig. 6d). Therefore, the Ugd1 function or the UGD1 expression was reduced in the suppressor containing strains which may result in the decrease of UDP-GlcUA accumulation.

UXS1 regulates intracellular 5-FC accumulation. It was not clear how accumulation of UDP-GlcUA could alter cryptoccal susceptibility to 5 -FC. One can speculate that a high concentration of UDP-GlcUA could affect the cellular intake of 5-FC. We determined the accumulation of tritium-labeled 5-FC in various strains through time course experiment. After incubation of cells in the presence of $\left[{ }^{3} \mathrm{H}\right]-5-\mathrm{FC}$ for $20 \mathrm{~min}$ or longer, all three types of WM276 uxs1 deletion mutants accumulated significantly lower amount of $\left[{ }^{3} \mathrm{H}\right]-5-\mathrm{FC}$ compared to the wild type (Fig. 7 C2053, WL8, and WL9 vs. WM276). When the UGD1 G19A variant was introduced into the genome of each uxs1 deletion mutant, the accumulation of $\left[{ }^{3} \mathrm{H}\right]-5-\mathrm{FC}$ increased significantly compared to that of each deletion strain (Fig. 7, C2104, C2143, and C2030). Similarly, the uxs $1 \Delta$ mutant of KN99a, 13C2, accumulated significantly lower levels of $\left[{ }^{3} \mathrm{H}\right]-5-F C$ than the wild-type strain. When either the $n r g 1$ deletion variant or the UGD1 ${ }^{G 19 A}$ variant was introduced into $13 \mathrm{C} 2$, the accumulation of $\left[{ }^{3} \mathrm{H}\right]-5-\mathrm{FC}$ was significantly increased compared to $13 \mathrm{C} 2$ (Fig. 7d, C2050 and C2119). These data indicate that UXS1 and its suppressors can regulate the intracellular accumulation of 5-FC both in C. neoformans and C. gattii.

UXS1 modulates the expression levels of the purine-cytosine permease. Since 5 -FC enters the cells primarily via the purinecytosine permease Fcy2 in many fungi and the cryptococcal $f c y 2 \Delta$ mutants were resistant to 5 -FC (Table 2), it was possible that the observed alteration of the 5-FC accumulation in uxs1 deletants was caused by modification of the cellular localization or expression levels of Fcy2. We tagged Fcy2 at its N-terminus with $\mathrm{mNG}$ and FLAG at the native FCY2 locus in WM276, uxs1 1 , and UGD1 ${ }^{G 19 A}$ suppressor strain respectively. The tagged strains, C2151, C2152, and $\mathrm{C} 2153$, had the same 5-FC resistance levels as their parental strains (Table 2) suggesting that N-terminal tagging of Fcy2 did not affect the function of Fcy2. Microscopic examinations revealed that the Fcy2 was mainly localized to the cell membranes a well as in the structures reminiscent of large vesicles in all three tagged strains (Fig. 8a). Additionally, the fluorescence intensity was slightly lower in the uxs $1 \Delta$ mutant compared to that of the wild type and the strain of $u x s 1 D$ containing UGD1 ${ }^{G 19 A}$ (Fig. 8b). Western blot analysis confirmed that Fcy2 protein levels were significantly lower in the uxs $1 \Delta$ mutant than the wild type and $U G D^{G 19 A}$ strains (Fig. 8c). These results indicated that the status of UXS1 and the presence of its suppressors regulated the accumulation of 5-FC inside the cells via modulation of the Fcy2 expression levels.

\section{Discussion}

Our study reveals that 5-FC induces various types of genetic modifications in both C. neoformans and C. gattii. Genetic modifications appeared to have been random event since various genomic variants were detected in those areas related as well as unrelated to 5-FC resistance. The high frequency of resistant mutants reported here has biological relevance since it implicates 5-FC to be mutagenic. The frequency of resistance far exceeds the classical spontaneous mutation frequency in eukaryotes and hence, it is unlikely that the resistant clones represent unusually large number of pre-existing spontaneous mutants. It is known that 5FC is converted to 5-fluorouracil inside the cells. 5-fluorouracil inhibits thymidylate synthase and thymidine starvation leads to single- and double-strand DNA breaks. Thymidine deprivation also leads to incorporation of uracil into DNA in place of thymidine and causes mutations in other eucaryotes ${ }^{38-40}$. 5-fluorouracil is massively incorporated into ribonucleic acid causing abnormal rRNA assembly, tRNA misfolding, and mRNA misreading ${ }^{41-44}$. It is possible that at moderate concentrations, 5-FC may not sufficiently inhibit cell growth and the metabolized 5-FC can cause various modifications including chromosome aneuploidy. Subsequently, cells containing modifications in genes relevant for 5-FC resistance are selected and form resistant colonies at high frequency. At high concentrations, however, 5-FC appears to inhibit the growth effectively and select out spontaneous mutants with genetic alterations in the genes relevant for 5-FC resistance. It is known that spontaneous mutation frequency can vary widely 
a
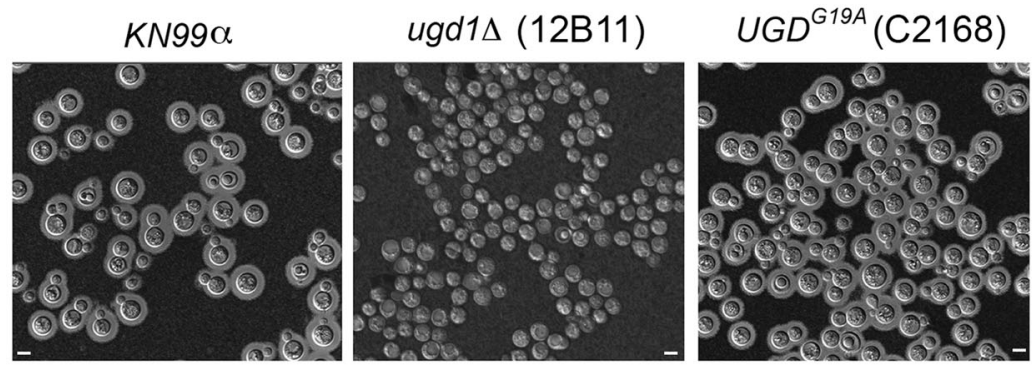

b

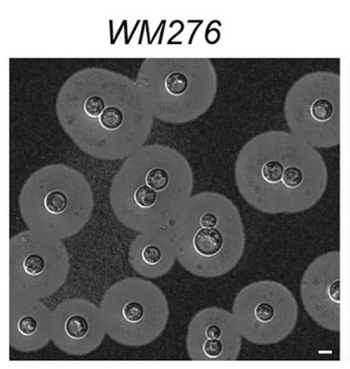

$U G D^{G 19 A}(\mathrm{C} 2061)$

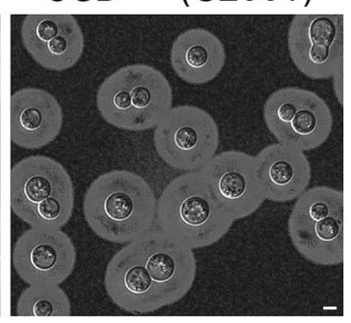

C
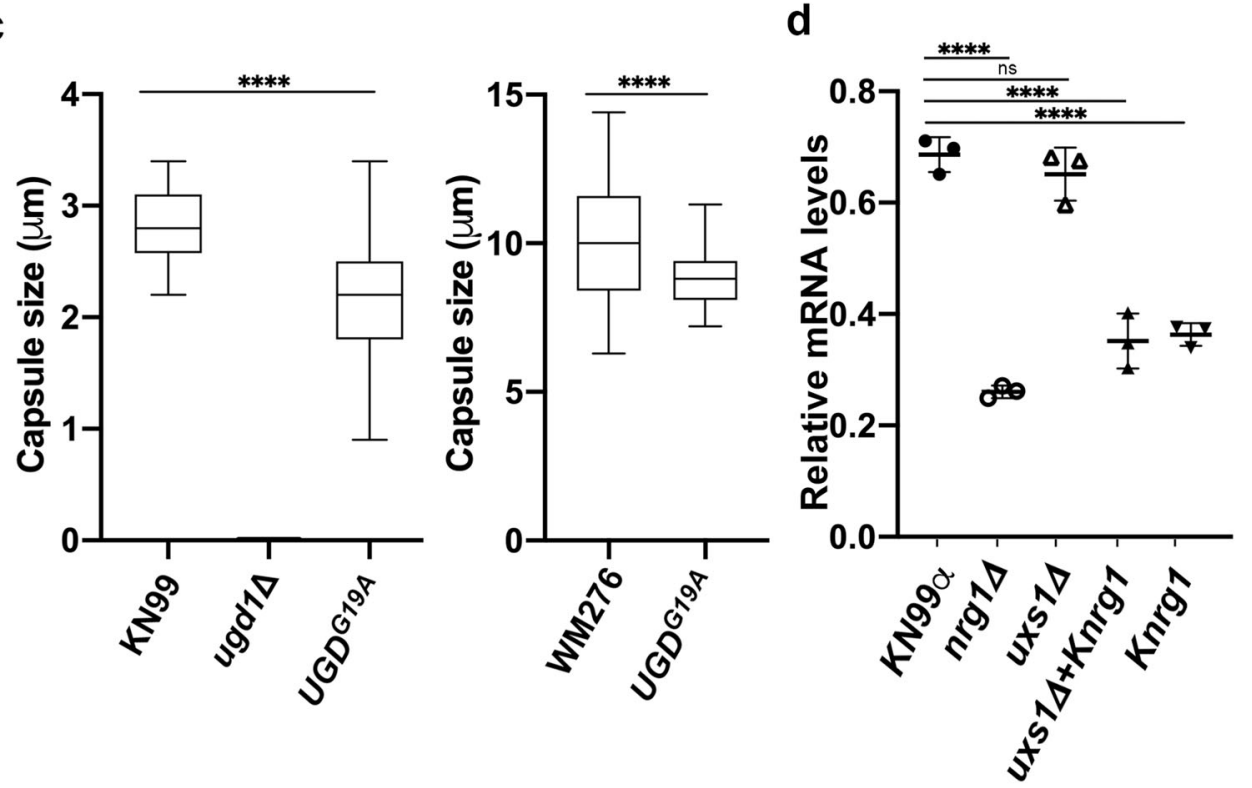

Fig. 6 The Ugd1 function or the UGD1 expression levels is affected in suppressor containing strains. a-c Capsule size is affected by the UGD1G19A mutation. Cells were stained by India ink and visualized by microscopy. The experiments were repeated 4 times independently with similar results. The capsule sizes of more than 65 cells were measured from 5 different fields for each indicated strain. Capsular thickness was defined as the distance between the visualized cell wall and the edge of the capsule. The box-and-whiskers plot is used to show median, quartiles (boxes), and range (whiskers). Two-tailed, unpaired t-test $\left({ }^{\star \star \star \star} p<0.0001\right)$. Bar $=5 \mu \mathrm{m}$. d UGD1 expression is downregulated in the Knrg1 containing strains. RNA was isolated and the expression levels of UGD1 were determined by qRT-PCR, normalized to the actin gene. The experiments were repeated three times. One-way ANOVA with Tukey's test for multiple comparisons (ns $=$ not significant and ${ }^{\star \star \star *} p<0.0001$ ).

depending on the strains within the same species. For example, mutation frequency to rifampicin resistance in Helicobacter pylori varied between $3 \times 10^{-5}$ and $4 \times 10^{-8}$ among tested strains ${ }^{45}$. In this study we identified at least 5 genes that contribute to 5 -FC resistance. At concentrations close to 400-fold of MIC, both C. neoformans (H99, $10 \mu \mathrm{g} / \mathrm{ml}$ ) and C. gattii (WM276, $5 \mu \mathrm{g} / \mathrm{ml}$ ) developed 5-FC resistant colonies at the frequency close to $1 \times 10^{-6}$ which is within the ranges of classical spontaneous mutation frequency. It is difficult to extrapolate our in vitro results in the clinical settings since the 5-FC dosage recommended for therapy is based on the body weight of patients while our study was conducted on agar plates at $30^{\circ} \mathrm{C}$. Although it has been recommended that the 5-FC dose in patients be adjusted to maintain the serum 5FC levels at 50 to $100 \mu \mathrm{g} / \mathrm{ml}^{46}$, possibility still exists that 5-FC can induce cryptococcal genomic changes in the host during long term treatment and cause therapeutical failure.

In understanding the regulation of 5-FC drug susceptibility in cryptococci, we identified several factors that contribute to the changes in susceptibility including mutations in FCY2, FCY1, FUR1, UXS1, and URA6 genes in addition to several unverified variants that remains to be explored; developing of suppressors; chromosome aneuploidy and the regulation of the gene on aneuploid chromosome. Aneuploidy is known to be associated with an adaptation process in pathogenic yeasts to fluconazole stress $25,47,48$. The most prevalent aneuploidy found in fluconazole heteroresistant pathogenic yeasts is the chromosome that harbors the genes encoding the target of azoles, the major efflux pump or transcription regulator of drug efflux pumps ${ }^{25,47}$. Our results 
a

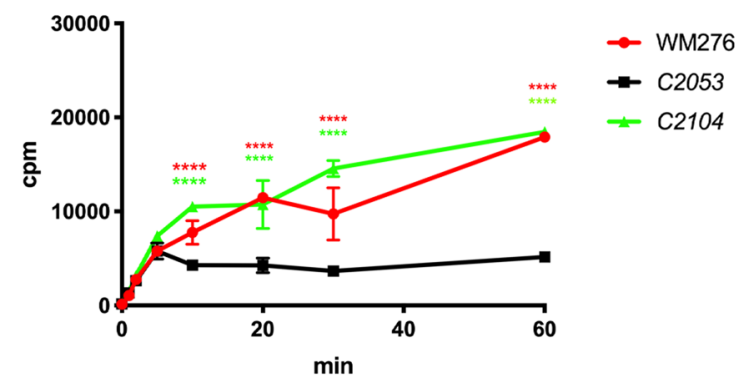

C

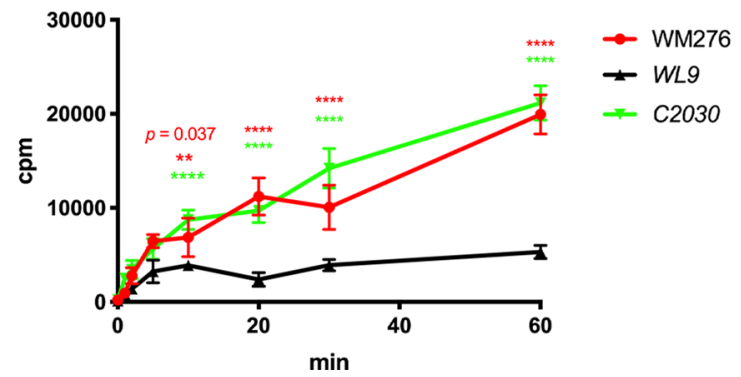

b

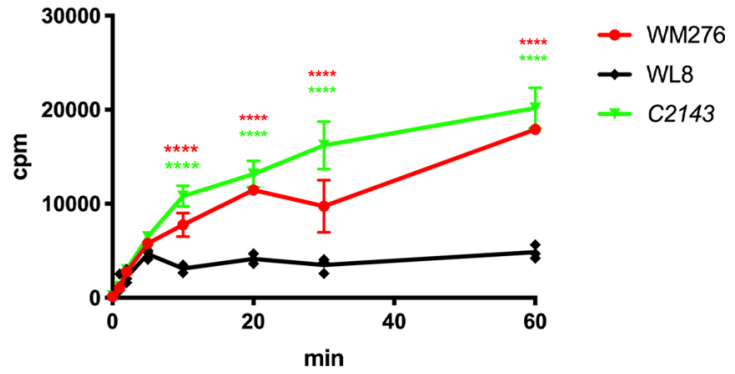

d

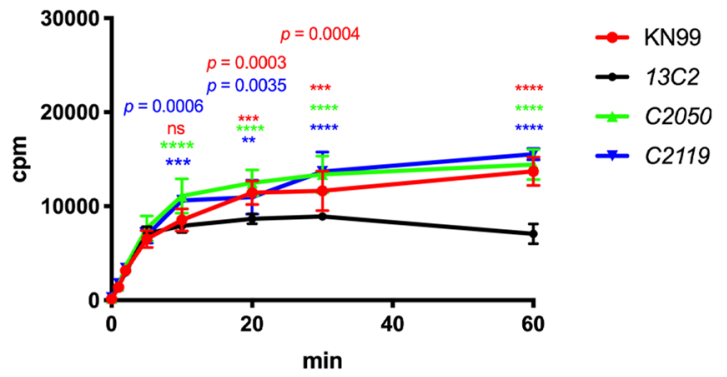

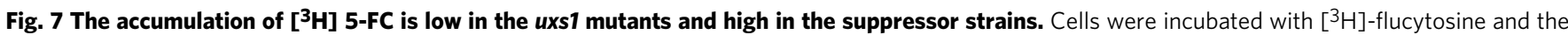
accumulation of $\left[{ }^{3} \mathrm{H}\right]$-flucytosine was determined at indicated time. The experiments were repeated three times and data are presented as mean values \pm standard deviation. Each panel contains the wild type, the uxs1 mutant and the suppressor strains derived from WM276 (a), WL8 (b), WL9 (c) and KN99 $\alpha$ (d). Two-way ANOVA with Tukey's multiple comparisons test was used to compare the differences at the indicated time point between the uxs1 mutant and the wild type or between the uxs1 mutant and the suppressor strain (ns = not significant, ${ }^{\star \star} p<0.0021,{ }^{\star \star \star} p<0.0002$, and ${ }^{\star \star \star \star} p<0.0001$ ).

indicated that chr1 aneuploidy was also beneficial for cryptococcal cells in responding to 5-FC stress. Interestingly, an extra copy of the $\mathrm{ABC}$ transporter gene, $A F R 1$, slightly increases the drug resistance levels in H99. Although Afr1 is known as a primary azole efflux pump and deletion of AFR1 has no clear impact on the level of 5-FC resistance ${ }^{49}$, Afr1 might have a supplementary minor role in transporting other xenobiotics such as 5FC in combination with other transporters. Thus, extra copy of AFR1 could contribute modestly to the 5 -FC resistance in H99. It is also interesting that extra copies of AFR1 slightly increased the resistance levels of 5-FC in R265 but not in WM276. We observed several other differences among the three strains analyzed. For instance, 5-FC MIC was higher in R265 compared to H99 and WM276 and R265 had a higher frequency of 5-FC resistant colonies than H99 and WM276 at similar folds of 5-FC MIC levels. Moreover, increased chromosome copy number was not found among the sequenced drug-resistant clones derived from R265 and the growth rate of the suppressor strains was improved in WM276 but not in KN99a background. Additionally, NRG1 variant was able to suppress 5 -FC resistance phenotype of the uxs1 mutant in C. neoformans, KN99a but not in C. gattii, WM276. These results indicate that the genetic wiring for 5-FC resistance is divergent between different strains of the cryptococcal species complex.

Isolation of suppressors for 5-FC resistant phenotype was serendipitous during repeated transfers of the usx 1 mutants in nonselective medium. The growth rate of the uxs1 mutants containing $U G D 1^{G 19 A}$ suppressor was improved in the WM276 background, which could have increased the chance of isolating suppressor strains. It is not clear how the $U G D 1^{G 19 A}$ suppressor influences the growth in WM276 background. Since the presence of the suppressors reduced the accumulation of UDP-GlcUA, it is possible that such a reduction could restore the growth defect of uxs1 1 mutant in WM276. In contrast, the growth rate was not improved in the suppressors containing KN99 a uxs $1 \Delta$ mutants. It is unclear how repeated transfers of the KN99 $u$ uxs $1 \Delta$ strains in drug-free media resulted in the selection for the suppressor containing cells with no growth rate advantage. One possible scenario can be that deletion of UXS1 in KN99a may reduce life span of the cells and the suppressor containing cells could overcome the deficiency. As a consequence, the uxs 1 mutant cells could have been outnumbered by the suppressor containing cells during repeated transfers.

It is not clear how exactly UDP-GlcUA accumulation might impact permease expression. It is possible that high levels of UDP-GlcUA might affect the expression of permease by altering the expression of transcription factor(s). However, we have failed to identify a probable transcription factor gene through analysis of transcriptome profile using the RNAs isolated from the wildtype, the WM276 uxs1 $1 \Delta$ mutant, and suppressor containing strains in the uxs $1 \Delta$ and wild-type background. It is also possible that accumulation of UDP-GlcUA might affect the Fcy2 levels at the translational or post-translational steps. The detailed mechanism(s) of how UDP-GlcUA might affect permease expression remains to be elucidated.

\section{Methods}

Strains and culture conditions. Strains relevant to the study are listed in Supplementary Table 1 . KN99a is derived from H99 ${ }^{50}$. Strains were stored in $25 \%$ glycerol stocks at $-80^{\circ} \mathrm{C}$ until use and were maintained on YPD ( $1 \%$ yeast extract, $2 \%$ peptone, $2 \%$ glucose) agar plates at $30^{\circ} \mathrm{C}$ for routine cultures. For the 5 -FC resistant clones, the cultures were revived on YNB (yeast nitrogen base without amino acids) agar medium supplemented with 5-FC at the concentrations from which the clones were originally isolated. For capsule detection, cells were grown in Dulbecco's modified Eagle's medium (Gibco Lab-oratories, GrandIsland, NY) with $25 \mathrm{mM}$ glucose $(4.5 \mathrm{~g} / \mathrm{l})$ supplemented with $22 \mathrm{mM} \mathrm{NaHCO}_{3}, 25 \mathrm{mM} \mathrm{NaMOPS} \mathrm{pH}$ 7.3 with $5 \% \mathrm{CO}_{2}$ at $37^{\circ} \mathrm{C}$ for $48 \mathrm{~h}$.

Determination of MIC for antifungal antibiotics. Unless specified, the 5-FC MICs were determined by using the Liofilchem ${ }^{\circledast}$ MTS $^{\mathrm{TM}}$ (MIC Test Strips) (Liofilchem, 
a
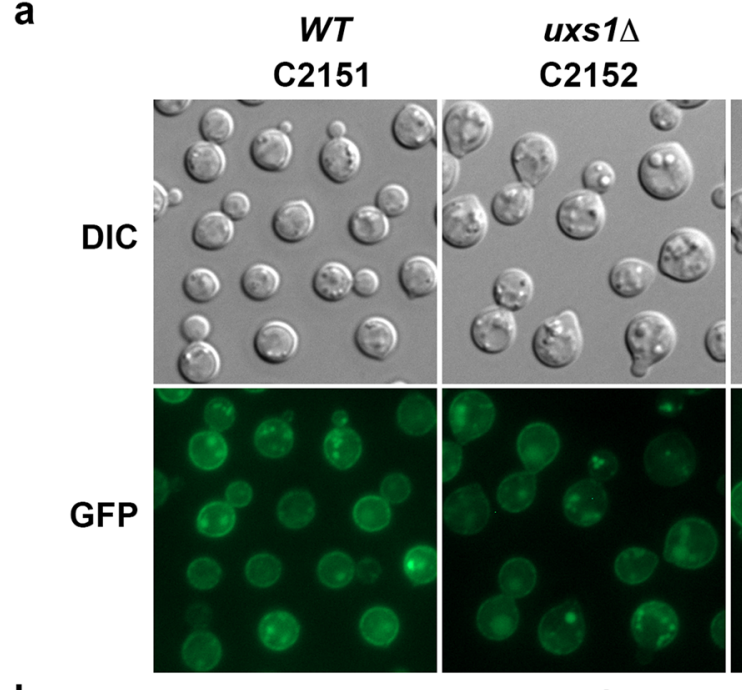

ugd1 ${ }^{G 19 A}$

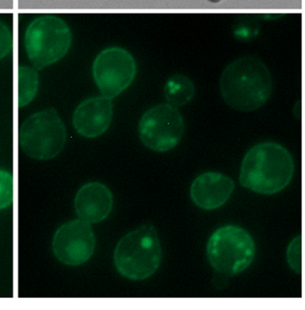

C2513

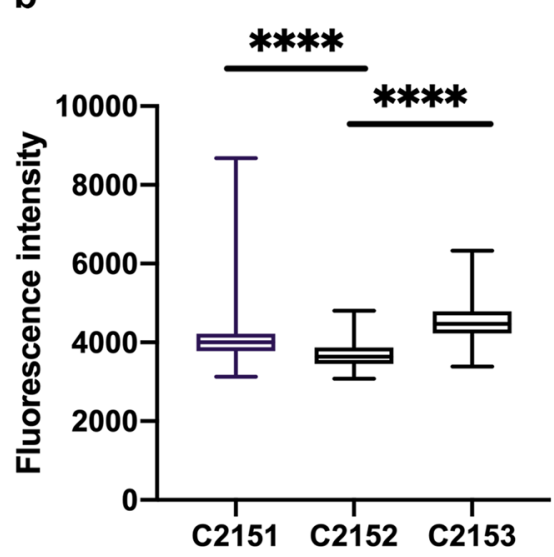

C
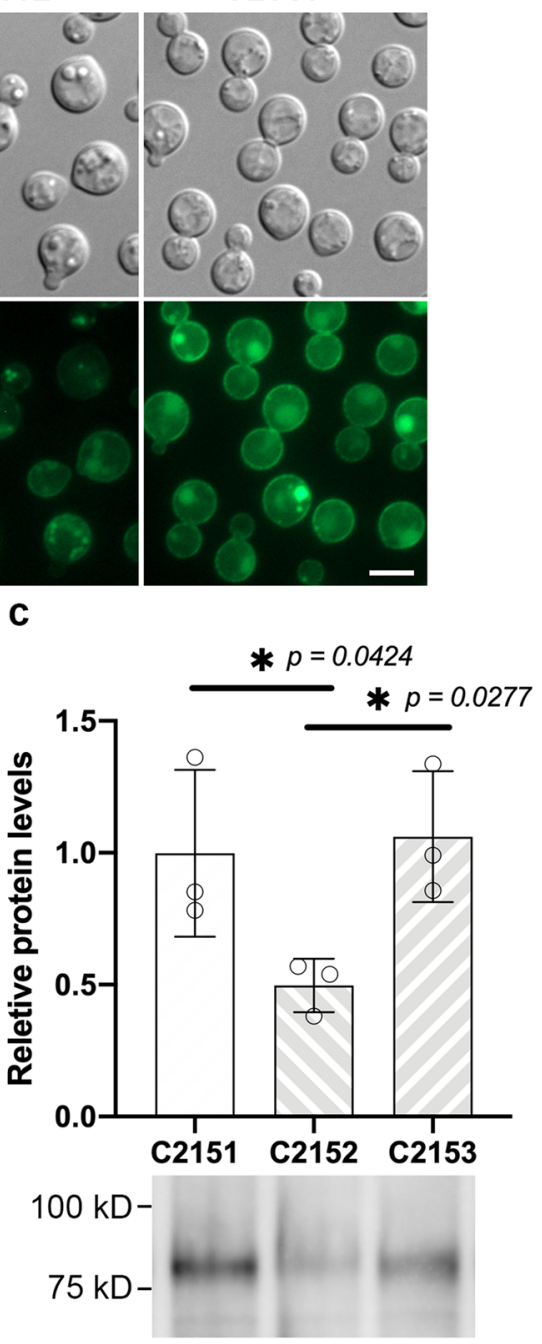

Fig. 8 Fcy2 protein levels are low in uxs1 mutants and high in the suppressor strains. a The localization of Fcy2 in the indicated strains was visualized by microscopy and photographed. Bar $=5 \mu \mathrm{m}$. $\mathbf{b}$ The mean fluorescence intensity of each strain from (a) were quantified from three separate images. The box-and-whiskers plot is used to show median, quartiles (boxes), and range (whiskers). One-way ANOVA with Tukey's test for multiple comparisons $\left.{ }^{\star \star \star \star} p<0.0001\right)$. c The relative amounts of the FLAG tagged Fcy2 was analyzed by western blot (bottom) and the expression levels of Fcy 2 were quantified by measuring the band intensity in the western blots, normalized to that of the total protein from stain-free blot and compared to the expression levels in C2151. For the full scan blots, see the Source data file. Data are presented as mean values \pm standard deviation from three biological repeats. One-way ANOVA with Tukey's test for multiple comparisons $\left({ }^{*} p<0.0332\right.$ and $\left.{ }^{* \star \star *} p<0.0001\right)$.

Waltham, MA) with modifications. Approximately $2 \times 10^{6}$ cells were plated on YNB agar plates followed by application of test strips. The plates were incubated at $30^{\circ} \mathrm{C}$ and photographed after $72 \mathrm{~h}$. We noted that the MIC results from Liofilchem ${ }^{\circledast}$ MTS $^{T M}$ were lower than the previously published results which was based on $A B$ Biodisk Etest strips ${ }^{\otimes 29}$. When MIC levels of mutant strains were close to the wild type, we used the broth MIC method recommended by CLSI M27-A3 ${ }^{51}$ to determine the MIC levels. Briefly, 5-FC or fluconazole was dispensed in 96-well microtiter plates with 2-fold serial dilutions of drug concentrations ranging from $32 \mu \mathrm{g} / \mathrm{ml}$ to $0.0625 \mu \mathrm{g} / \mathrm{ml}$ in MOPS-buffered RPMI 1640 and incubated at $37^{\circ} \mathrm{C}$ for $72 \mathrm{~h}$.

Determination of the 5-FC resistance frequency. Three days old cells grown on YPD plate were harvested and plated on YNB media supplemented with 5-FC at various concentrations which corresponded to approximately $20-, 40-, 100-$, and 400 -fold of 5-FC MIC of each tested strain. Plates were incubated at $30^{\circ} \mathrm{C}$ for 7 days and photographed with ChemiDoc MP using Image Lab software (Bio-Rad, Hercules, CA). Because the size of some of the 5-FC resistant colonies varied, we used CellProfiler 3.1.5 software $^{52}$ to unbiasedly determine the number of 5-FC resistant colonies in the photograph. We considered the colonies with minimal diameter greater than 8 pixels as the 5-FC resistant clones in all concentrations of 5-FC. The frequency of 5-FC resistance was calculated by dividing the number of the resistant colonies obtained from CellProfiler by the total number of the input cells.
Stability test of 5-FC resistant clones. The clones used for stability test were originally isolated from plates containing $0.5 \mu \mathrm{g} / \mathrm{ml} 5$ - $\mathrm{FC}$ for $\mathrm{H} 99$ (approximately 20fold of MIC) as well as WM276 (approximately 40 -fold of MIC) and $5 \mu \mathrm{g} / \mathrm{ml} \mathrm{5-FC}$ for R265 ( 100-fold MIC). The 5-FC resistant clones were grown in $2 \mathrm{ml}$ of YPD liquid media at a $30^{\circ} \mathrm{C}$ shaker incubator and $20 \mu \mathrm{l}$ of the culture was transferred to fresh YPD media daily for up to 51 days. At each indicated period during the daily transfer, cells from the YPD liquid culture were diluted and plated out on 5-FC plates and the percentage of the 5-FC resistant colonies was determined. For simplicity, the resistance frequency at or greater than $100 \%$ is expressed as $100 \%$.

Whole-genome sequencing. The 5-FC resistant clones were individually expanded by culturing for 3 days at $30^{\circ} \mathrm{C}$ on YNB plate supplemented with 5-FC at the concentrations in which the clones were originally isolated. For the 5-FC susceptible clones, individual clones were grown on YPD plate at $30^{\circ} \mathrm{C}$ for 2 days. Cells were harvested from the plates and lyophilized. Genomic DNA was isolated using the cetyltrimethylammonium bromide (CTAB) extraction method ${ }^{53}$ with modifications. Paired-end libraries ( $150 \mathrm{bp}$ ) were prepared and sequenced using an Illumina HiSeq 4000 platform (Novogene, Davis, CA). The analysis was generated using Partek ${ }^{\oplus}$ Flow $^{\circledast}$ software, version 7.0, 2017 (Partek Inc., St. Louis, MO). Illumina reads were trimmed from both end with minimal quality level at 38 and aligned to the reference genome of H99 (CNA3), R265 (V2) and WM276 
(ASM18594v1), respectively, using BWA. Genome-wide copy number was determined by CNVkit with WGS method and the results were used to generate heatmap with $\mathrm{CNVkit}^{54}$. SNP and indel calling were performed using FreeBayes variant caller with haploid setting. The data was filtered by allele frequency greater than 4.5 and read depth greater than 5. FungiDB was also used to determine putative function and orthology of the genes which contained features called variants in the dataset ${ }^{55}$

Gene manipulation. Standard molecular cloning combined with overlap PCR and In-Fusion ${ }^{\oplus}$ HD Cloning kit (Clontech, Mountain View, CA) were used to build the desired constructs. To generate the construct of each variant, primers carrying desired modifications (Supplementary Table 2) were used for PCR amplification and cloned into pCR2.1 vector (Invitrogen, Carlsbad, CA) along with indicated selectable markers. To make Xnrg1, the $3^{\prime}$-end of NRG1 in Wnrg1 was digested with NheI and Bsu36I and replaced with the NheI/Bsu36I fragment derived from Knrg1. Gene deletion, complementation, tagging, and allele replacement were obtained by biolistic transformation. Homologous integration was confirmed by PCR and Southern blot analysis.

The AFR1 gene was amplified by PCR from H99, cloned into pCR2.1 along with the $N E O$ selectable maker and inserted in the intergenic region between CNAG_03012 and CNAG_03013 of Chr3 ${ }^{25}$ to generate pYCC1200. To construct the strains containing an extra copy of AFR1 in chr3 of H99, pYCC1200 was transformed into $\mathrm{H} 99$ and the corrected clones were identified by PCR and Southern blot analysis. pYCC1200 was also transformed into R265 and WM276 to generate strains contained extra copies of $A F R 1$ at ectopic sites.

Determination of growth rate. The growth curves for each strain were obtained by using 48 wells microtiter plates (Costa 3548, Mesa, CA) with Synergy H1 plate reader (Biotek, Winooski, VT). Cells were grown in $5 \mathrm{ml} \mathrm{YPD} \mathrm{for} 22-24 \mathrm{~h}$ at $30^{\circ} \mathrm{C}$ with shaking. The culture was diluted to $\mathrm{OD}=0.05$ in YPD and $500 \mu \mathrm{l}$ of cells was applied to each well. For each strain, 7 wells were used and one well containing only YPD was used as a control. Plate was sealed with Breathe-Easy film (Diversified Biotch, Boston, USA) and placed in the plate reader at $30^{\circ} \mathrm{C}$. The program setting was double orbital, $2 \mathrm{~mm}$ fast with $548 \mathrm{cpm}$ and the reading was taken at 15 min intervals for $24 \mathrm{~h}$. The growth rate was obtained from at least three independent experiments. The Max V was obtained from log phase using Gen5 Data Analysis Software (Biotek, Winooski, VT) and the doubling time was calculated from Max V.

Isolation of nucleotide sugar. Nucleotide sugars were analyzed from total protein extracts ${ }^{32}$. Samples were analyzed by a Thermo Scientific Vanquish UPLC coupled with a Q-Exactive high resolution accurate-mass spectrometer (HRAM-MS, Thermo Scientific, Waltham, MA) with heated electrospray ionization (HESI-II) in negative ion mode. $50 \mu \mathrm{l}$ of $2 \mu \mathrm{g} / \mu \mathrm{l}$ protein samples were added to $200 \mu \mathrm{l}$ internal standard solution containing $100 \mathrm{ng} / \mathrm{ml}$ of ${ }^{13} \mathrm{C}_{6}$-Saccharin in acetonitrile, vortexed for $5 \mathrm{~min}$, and then centrifuged at $4^{\circ} \mathrm{C}, 20,000 \times g$ for $15 \mathrm{~min}$. $200 \mu \mathrm{l}$ supernatant was transferred into a LC-MS vial, and mixed with $200 \mu \mathrm{L}$ of $\mathrm{H}_{2} \mathrm{O}$ for analysis. An Agilent Infinitylab Poroshell 120 EC-C18 column $(2.1 \mathrm{~mm} \times 100 \mathrm{~mm}, 1.9 \mu \mathrm{m})$ was used. Column was maintained at $40^{\circ} \mathrm{C}$ and samples were kept in the autosampler at $4{ }^{\circ} \mathrm{C}$. The injection volume was $3 \mu \mathrm{l}$. Chromatographic conditions were as follows: Solvent A: $5 \% \mathrm{MeOH}, 3.5 \mathrm{mM}$ dibutylamine, pH 5.5 (adjust with $\mathrm{AcOH}$ ), Solvent B: 62\% ACN, 33\% IPA, 5\% H2O, $3.5 \mathrm{mM}$ dibutylamine, pH 5.5 (adjust with $\mathrm{AcOH}$ ). The flow rate was $225 \mu \mathrm{l} / \mathrm{min}$, and the gradient was $10 \% \mathrm{~B}$ at $0 \mathrm{~min}$ for $0.15 \mathrm{~min}$, increased to $100 \% \mathrm{~B}$ at $3 \mathrm{~min}$, remained at $100 \% \mathrm{~B}$ until $4.5 \mathrm{~min}$, returned to $10 \%$ B at $5.5 \mathrm{~min}$. The total running time was $10 \mathrm{~min}$. Samples were analyzed in triplicates and three biological repeats were performed for each strain. Quantitation was based on the $m / z, 565.0489$ for UDP-glucose, 535.0372 for UDPxylose, 579.0276 for UDP-glucuronic acid, and 188.0116 for ${ }^{13} \mathrm{C}_{6}$-Saccharin and expressed as relative levels to the internal standard ${ }^{13} \mathrm{C}_{6}$-Saccharin.

Microscopy. Zeiss observer fluorescent microscope equipped with an AxioCam digital camera was used to take differential interference contrast microscopy (DIC) and fluorescent images. The exposure times and other microscope settings were held constant between compared images. We employed the Surface function in the Imaris software (Bitplane Scientific Software, USA) to quantify the fluorescence intensity of cells expressing Fcy2-mNG. The fluorescence intensity was measured from three different images of each strains and at least 275 cells of each strain were evaluated. The intensity mean of each cell was imported into Graph Pad Prism (version 8.4.3) and analyzed.

5-FC accumulation assay. The cells were grown in YPD to log phase, washed and then transferred to YNB media and incubated for additional $3 \mathrm{~h}$ at $30^{\circ} \mathrm{C}$. Cells were harvested, washed with cold YNB without glucose and adjusted to $10 \mathrm{OD} / \mathrm{ml}$. Each $100 \mu \mathrm{l}$ of cells were mixed with $100 \mu \mathrm{l}$ of $2 \times$ YNB media with glucose supplemented with $200 \mu \mathrm{M}$ flucytosine and $0.8 \mu \mathrm{Ci}$ of $\left[{ }^{3} \mathrm{H}\right.$ ] flucytosine (Moravek Inc; $4.0 \mathrm{Ci}$ / mmole, $1 \mathrm{mCi} / \mathrm{ml}$ ). Cells were incubated at $30^{\circ} \mathrm{C}$ in a water-bath shaker and $50 \mu \mathrm{l}$ aliquots of sample were removed at $0,1,2,5,10,20,30$, and $60 \mathrm{~min}$ and added to 2 $\mathrm{mL}$ cold media containing $20 \mathrm{mM}$ flucytosine. Cells were immediately filtered with
$24 \mathrm{~mm}$ Whatman GF/C filter and washed with cold YNB-A-N and assayed for radioactivity.

Protein extraction and immunoblot analysis. Total proteins were extracted with $\mathrm{NaOH}$ method $^{27}$. An equal amount of protein $(20 \mu \mathrm{g})$ was loaded and run on the Any kD Criterion TGX Stain-Free gel (Bio-Rad, Richmond, CA). The western blot was incubated with anti-FLAG antibody and developed using the Clarity Western ECL (Bio-Rad, Richmond, CA). The signal was quantitated using ChemiDoc MP imaging system (Bio-Rad, Richmond, CA) and band intensities were normalized to stain-free blots to control for loading.

Statistical analysis. Statistical analyses were performed using Graph Pad Prism (version 8.4.3). Student's $t$ test and ANOVA followed by multiple-comparison test were used to determine whether there were significant differences between indicated samples.

Reporting summary. Further information on research design is available in the Nature Research Reporting Summary linked to this article.

\section{Data availability}

The sequencing data from this study have been submitted to the NCBI BioProject under the accession PRJNA704667. FungiDB is available at [https://fungidb.org/fungidb/app]. All relevant data are available upon request. Source data are provided with this paper.

Received: 19 January 2021; Accepted: 14 May 2021; Published online: 08 June 2021

\section{References}

1. Duschinsky, R., Pleven, E. \& Heidelberger, C. The synthesis of 5-fluoropyrimidines. J. Am. Chem. Soc. 79, 4559-4560 (1957)

2. Polak, A. \& Grenson, M. Evidence for a common transport system for cytosine, adenine and hypoxanthine in Saccharomyces cerevisiae and Candida albicans. Eur. J. Biochem. 32, 276-282 (1973).

3. Paluszynski, J. P., Klassen, R., Rohe, M. \& Meinhardt, F. Various cytosine/ adenine permease homologues are involved in the toxicity of 5-fluorocytosine in Saccharomyces cerevisiae. Yeast 23, 707-715 (2006).

4. Edlind, T. D. \& Katiyar, S. K. Mutational analysis of flucytosine resistance in Candida glabrata. Antimicrob. Agents Chemother. 54, 4733-4738 (2010).

5. Ghannoum, M. A. \& Rice, L. B. Antifungal agents: mode of action, mechanisms of resistance, and correlation of these mechanisms with bacterial resistance. Clin. Microbiol Rev. 12, 501-517 (1999).

6. Hope, W. W., Tabernero, L., Denning, D. W. \& Anderson, M. J. Molecular mechanisms of primary resistance to flucytosine in Candida albicans. Antimicrob. Agents Chemother. 48, 4377-4386 (2004)

7. Espinel-Ingroff, A. Mechanisms of resistance to antifungal agents: yeasts and filamentous fungi. Rev. Iberoam. Micol. 25, 101-106 (2008).

8. Waldorf, A. R. \& Polak, A. Mechanisms of action of 5-fluorocytosine. Antimicrob. Agents Chemother. 23, 79-85 (1983).

9. Kwon-Chung, K. J. et al. Cryptococcus neoformans and Cryptococcus gattii, the etiologic agents of cryptococcosis. Cold Spring Harb. Perspect. Med. 4, a019760 (2014).

10. Perfect, J. R. et al. Clinical practice guidelines for the management of cryptococcal disease: 2010 update by the infectious diseases society of america. Clin. Infect. Dis. 50, 291-322 (2010).

11. Masur, H. et al. Prevention and treatment of opportunistic infections in HIVinfected adults and adolescents: updated guidelines from the Centers for Disease Control and Prevention, National Institutes of Health, and HIV Medicine Association of the Infectious Diseases Society of America. Clin. Infect. Dis. 58, 1308-1311 (2014).

12. Hospenthal, D. R. \& Bennett, J. E. Flucytosine monotherapy for cryptococcosis. Clin. Infect. Dis. 27, 260-264 (1998).

13. Block, E. R., Jennings, A. E. \& Bennett, J. E. 5-fluorocytosine resistance in Cryptococcus neoformans. Antimicrob. Agents Chemother. 3, 649-656 (1973).

14. Houst, J., Spizek, J. \& Havlicek, V. Antifungal drugs. Metabolites 10, 106 (2020).

15. Papon, N. et al. Molecular mechanism of flucytosine resistance in Candida lusitaniae: contribution of the FCY2, FCY1, and FUR1 genes to 5-fluorouracil and fluconazole cross-resistance. Antimicrob. Agents Chemother. 51, 369-371 (2007).

16. Whelan, W. L. The genetic basis of resistance to 5-fluorocytosine in Candida species and Cryptococcus neoformans. Crit. Rev. Microbiol 15, 45-56 (1987).

17. Gsaller, F. et al. Mechanistic Basis of pH-Dependent 5-Flucytosine Resistance in Aspergillus fumigatus. Antimicrob Agents Chemother. https://doi.org/ 10.1128/AAC.02593-17 (2018). 
18. Costa, C. et al. New mechanisms of flucytosine resistance in C. glabrata unveiled by a chemogenomics analysis in S. cerevisiae. PLoS ONE 10, e0135110 (2015).

19. Billmyre, R. B., Applen Clancey, S., Li, L. X., Doering, T. L. \& Heitman, J. 5fluorocytosine resistance is associated with hypermutation and alterations in capsule biosynthesis in Cryptococcus. Nat. Commun. 11, 127 (2020).

20. Janbon, G. et al. Analysis of the genome and transcriptome of Cryptococcus neoformans var. grubii reveals complex RNA expression and microevolution leading to virulence attenuation. PLoS Genet. 10, e1004261 (2014).

21. Kidd, S. E. et al. A rare genotype of Cryptococcus gattii caused the cryptococcosis outbreak on Vancouver Island (British Columbia, Canada). Proc. Natl Acad. Sci. USA 101, 17258-17263 (2004).

22. D'Souza, C. A. et al. Genome variation in Cryptococcus gattii, an emerging pathogen of immunocompetent hosts. mBio 2, e00342-00310 (2011).

23. Mondon, P. et al. Heteroresistance to fluconazole and voriconazole in Cryptococcus neoformans. Antimicrob. Agents Chemother. 43, 1856-1861 (1999).

24. Varma, A. \& Kwon-Chung, K. J. Heteroresistance of Cryptococcus gattii to fluconazole. Antimicrob. Agents Chemother. 54, 2303-2311 (2010).

25. Sionov, E., Lee, H., Chang, Y. C. \& Kwon-Chung, K. J. Cryptococcus neoformans overcomes stress of azole drugs by formation of disomy in specific multiple chromosomes. PLoS Pathog. 6, e1000848 (2010).

26. Stone, N. R. et al. Dynamic ploidy changes drive fluconazole resistance in human cryptococcal meningitis. J. Clin. Invest. 129, 999-1014 (2019).

27. Chang, Y. C., Khanal Lamichhane, A. \& Kwon-Chung, K. J. Cryptococcus neoformans, unlike Candida albicans, forms aneuploid clones directly from uninucleated cells under fluconazole stress. mBio https://mbio.asm.org/ content/9/6/e01290-18 (2018).

28. $\mathrm{Vu}, \mathrm{K}$. et al. Flucytosine resistance in Cryptococcus gattii is indirectly mediated by the FCY2-FCY1-FUR1 pathway. Med Mycol. 56, 857-867 (2018).

29. Khanal Lamichhane, A. et al. A Novel role of fungal type I myosin in regulating membrane properties and its association with d-amino acid utilization in Cryptococcus gattii. mBio https://mbio.asm.org/content/10/4/ e01867-19 (2019).

30. Liljelund, P. \& Lacroute, F. Genetic characterization and isolation of the Saccharomyces cerevisiae gene coding for uridine monophosphokinase. Mol. Gen. Genet. 205, 74-81 (1986).

31. Araki, H., Hamatake, R. K., Johnston, L. H. \& Sugino, A. DPB2, the gene encoding DNA polymerase II subunit B, is required for chromosome replication in Saccharomyces cerevisiae. Proc. Natl Acad. Sci. USA 88, 4601-4605 (1991).

32. Griffith, C. L., Klutts, J. S., Zhang, L., Levery, S. B. \& Doering, T. L. UDPglucose dehydrogenase plays multiple roles in the biology of the pathogenic fungus Cryptococcus neoformans. J. Biol. Chem. 279, 51669-51676 (2004)

33. Moyrand, F. \& Janbon, G. UGD1, encoding the Cryptococcus neoformans UDP-glucose dehydrogenase, is essential for growth at 37 degrees $\mathrm{C}$ and for capsule biosynthesis. Eukaryot. Cell 3, 1601-1608 (2004).

34. Moyrand, F., Klaproth, B., Himmelreich, U., Dromer, F. \& Janbon, G. Isolation and characterization of capsule structure mutant strains of Cryptococcus neoformans. Mol. Microbiol. 45, 837-849 (2002).

35. Bar-Peled, M., Griffith, C. L. \& Doering, T. L. Functional cloning and characterization of a UDP- glucuronic acid decarboxylase: the pathogenic fungus Cryptococcus neoformans elucidates UDP-xylose synthesis. Proc. Natl Acad. Sci. USA 98, 12003-12008 (2001).

36. Chen, P., Wang, D., Chen, H., Zhou, Z. \& He, X. The nonessentiality of essential genes in yeast provides therapeutic insights into a human disease. Genome Res. 26, 1355-1362 (2016).

37. Cramer, K. L., Gerrald, Q. D., Nichols, C. B., Price, M. S. \& Alspaugh, J. A. Transcription factor Nrg1 mediates capsule formation, stress response, and pathogenesis in Cryptococcus neoformans. Eukaryot. Cell 5, 1147-1156 (2006).

38. Adelberg, E. A. \& Coughlin, C. A. Bacterial mutation induced by thymine starvation. Nature 178, 531-532 (1956).

39. Domingo-Calap, P., Pereira-Gomez, M. \& Sanjuan, R. Nucleoside analogue mutagenesis of a single-stranded DNA virus: evolution and resistance. J. Virol. 86, 9640-9646 (2012).

40. Ahmad, S. I., Kirk, S. H. \& Eisenstark, A. Thymine metabolism and thymineless death in prokaryotes and eukaryotes. Annu Rev. Microbiol 52, 591-625 (1998).

41. Rosen, B., Rothman, F. \& Weigert, M. G. Miscoding caused by 5-fluorouracil. J. Mol. Biol. 44, 363-375 (1969).

42. Horowitz, J. \& Chargaff, E. Massive incorporation of 5-fluorouracil into a bacterial ribonucleic acid. Nature 184, 1213-1215 (1959).

43. Hills, D. C. \& Horowitz, J. Ribosome synthesis in Escherichia coli treated with 5-fluorouracil. Biochemistry 5, 1625-1632 (1966).

44. Lowrie, R. J. \& Bergquist, P. L. Transfer ribonucleic acids from Escherichia coli treated with 5-fluorouracil. Biochemistry 7, 1761-1770 (1968).

45. Bjorkholm, B. et al. Mutation frequency and biological cost of antibiotic resistance in Helicobacter pylori. Proc. Natl Acad. Sci. USA 98, 14607-14612 (2001).
46. Stamm, A. M. et al. Toxicity of amphotericin B plus flucytosine in 194 patients with cryptococcal meningitis. Am. J. Med. 83, 236-242 (1987).

47. Selmecki, A., Forche, A. \& Berman, J. Aneuploidy and isochromosome formation in drug-resistant Candida albicans. Science 313, 367-370 (2006).

48. Berman, J. Ploidy plasticity: a rapid and reversible strategy for adaptation to stress. FEMS Yeast Res. 16, fow020 (2016).

49. Chang, M., Sionov, E., Khanal Lamichhane, A., Kwon-Chung, K. J. \& Chang Y. C. Roles of Three Cryptococcus neoformans and Cryptococcus gattii Efflux Pump-Coding Genes in Response to Drug Treatment. Antimicrob. Agents Chemother. https://doi.org/10.1128/AAC.01751-17 (2018).

50. Nielsen, K. et al. Sexual cycle of Cryptococcus neoformans var. grubii and virulence of congenic a and alpha isolates. Infect. Immun. 71, 4831-4841 (2003).

51. Wayne, P. Reference Method for Broth Dilution Antifungal Susceptibility Testing of Yeasts-Third Edition:Approved Standard M27-A3. CLSI. National Committee for Clinical Laboratory Standards (2008).

52. Carpenter, A. E. et al. CellProfiler: image analysis software for identifying and quantifying cell phenotypes. Genome Biol. 7, R100 (2006).

53. Fujimura, H. \& Sakuma, Y. Simplified isolation of chromosomal and plasmid DNA from yeasts. Biotechniques 14, 538-540 (1993).

54. Talevich, E., Shain, A. H., Botton, T. \& Bastian, B. C. CNVkit: genome-wide copy number detection and visualization from targeted DNA sequencing. PLoS Comput Biol. 12, e1004873 (2016).

55. Stajich, J. E. et al. FungiDB: an integrated functional genomics database for fungi. Nucleic Acids Res. 40, D675-D681 (2012).

\section{Acknowledgements}

We thank Kazuhiro Ohshima and Nathan Kwon for technical assistance. The authors thank Brigit Shea Sullivan of NIH Library Editing Service, for manuscript editing assistance. Deletion strains of KN99 $\alpha$ and H99 were obtained from the Fungal Genetics Stock Center (Manhattan, Kansas, USA). This work was supported by the Division of Intramural Research (DIR), NIAID, NIH.

\section{Author contributions}

J.K.-C. and J.E.B. initiated the project. Y.C.C. designed the experiments. Y.C.C. and A.K.L performed the experiments. H.C. and P.J.W. performed the nucleotide sugar measurements. Y.C.C. and J.K-C wrote the manuscript. All authors reviewed the manuscript.

\section{Funding}

Open Access funding provided by the National Institutes of Health (NIH).

\section{Competing interests}

The authors declare no competing interests.

\section{Additional information}

Supplementary information The online version contains supplementary material available at https://doi.org/10.1038/s41467-021-23745-1.

Correspondence and requests for materials should be addressed to Y.C.C.

Peer review information Nature Communications thanks Marie Desnos-Ollivier and the other, anonymous, reviewers for their contribution to the peer review of this work. Peer reviewer reports are available.

Reprints and permission information is available at http://www.nature.com/reprints

Publisher's note Springer Nature remains neutral with regard to jurisdictional claims in published maps and institutional affiliations.

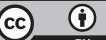

Open Access This article is licensed under a Creative Commons Attribution 4.0 International License, which permits use, sharing, adaptation, distribution and reproduction in any medium or format, as long as you give appropriate credit to the original author(s) and the source, provide a link to the Creative Commons license, and indicate if changes were made. The images or other third party material in this article are included in the article's Creative Commons license, unless indicated otherwise in a credit line to the material. If material is not included in the article's Creative Commons license and your intended use is not permitted by statutory regulation or exceeds the permitted use, you will need to obtain permission directly from the copyright holder. To view a copy of this license, visit http://creativecommons.org/ licenses/by/4.0/.

This is a U.S. Government work and not under copyright protection in the US; foreign copyright protection may apply 2021 